\title{
ON DIOPHANTINE EXPONENTS FOR LAURENT SERIES OVER A FINITE FIELD
}

\author{
TOMOHIRO OOTO
}

\begin{abstract}
In this paper, we study the properties of Diophantine exponents $w_{n}$ and $w_{n}^{*}$ for Laurent series over a finite field. We prove that for an integer $n \geq 1$ and a rational number $w>2 n-1$, there exist a strictly increasing sequence of positive integers $\left(k_{j}\right)_{j \geq 1}$ and a sequence of algebraic Laurent series $\left(\xi_{j}\right)_{j \geq 1}$ such that $\operatorname{deg} \xi_{j}=p^{k_{j}}+1$ and

$$
w_{1}\left(\xi_{j}\right)=w_{1}^{*}\left(\xi_{j}\right)=\ldots=w_{n}\left(\xi_{j}\right)=w_{n}^{*}\left(\xi_{j}\right)=w
$$

for any $j \geq 1$. For each $n \geq 2$, we give explicit examples of Laurent series $\xi$ for which $w_{n}(\xi)$ and $w_{n}^{*}(\xi)$ are different.
\end{abstract}

\section{INTRODUCTION}

Mahler 20 and Koksma [18 introduced Diophantine exponents which measure the quality of approximation to real numbers. Using the Diophantine exponents, they classified the set $\mathbb{R}$ all of real numbers. Let $\xi$ be a real number and $n \geq 1$ be an integer. We denote by $w_{n}(\xi)$ the supremum of the real numbers $w$ which satisfy

$$
0<|P(\xi)| \leq H(P)^{-w}
$$

for infinitely many integer polynomials $P(X)$ of degree at most $n$. Here, $H(P)$ is defined to be the maximum of the absolute values of the coefficients of $P(X)$. We denote by $w_{n}^{*}(\xi)$ the supremum of the real numbers $w^{*}$ which satisfy

$$
0<|\xi-\alpha| \leq H(\alpha)^{-w^{*}-1}
$$

for infinitely many algebraic numbers $\alpha$ of degree at most $n$. Here, $H(\alpha)$ is equal to $H(P)$, where $P(X)$ is the minimal polynomial of $\alpha$ over $\mathbb{Z}$.

We recall some results on Diophantine exponents. It is clear that $w_{1}(\xi)=w_{1}^{*}(\xi)$ for all real numbers $\xi$. Roth [29] established that $w_{1}(\xi)=w_{1}^{*}(\xi)=1$ for all irrational algebraic real numbers $\xi$. Furthermore, it follows from the Schmidt Subspace Theorem that

$$
w_{n}(\xi)=w_{n}^{*}(\xi)=\min \{n, d-1\}
$$

for all $n \geq 1$ and algebraic real numbers $\xi$ of degree $d$. It is known that

$$
0 \leq w_{n}(\xi)-w_{n}^{*}(\xi) \leq n-1
$$

for all $n \geq 1$ and real numbers $\xi$ (see Section 3.4 in [4]). Sprindžuk [32] proved that $w_{n}(\xi)=w_{n}^{*}(\xi)=n$ for all $n \geq 1$ and almost all real numbers $\xi$. Baker [3] proved that for $n \geq 2$, there exists a real number $\xi$ for which $w_{n}(\xi)$ and $w_{n}^{*}(\xi)$ are different. More precisely, he proved that the set of all values taken by the function $w_{n}-w_{n}^{*}$ contains the set $[0,(n-1) / n]$ for $n \geq 2$. In recent years, this result has been improved. Bugeaud [10, 5 ] showed that the set of all values taken by $w_{2}-w_{2}^{*}$ is equal to the closed interval $[0,1]$ and the set of all values taken by $w_{3}-w_{3}^{*}$ contains the set $[0,2)$. Bugeaud and Dujella

2010 Mathematics Subject Classification. 11J82, 11J61, 11J70.

Key words and phrases. Diophantine approximation, Diophantine exponent, Laurent series over a finite field. 
[8] proved that for any $n \geq 4$, the set of all values taken by $w_{n}-w_{n}^{*}$ contains the set $\left[0, \frac{n}{2}+\frac{n-2}{4(n-1)}\right)$.

Let $p$ be a prime. We can define Diophantine exponents $w_{n}$ and $w_{n}^{*}$ over the field $\mathbb{Q}_{p}$ of $p$-adic numbers in a similar way to the real case. Analogues of the above results for $p$-adic numbers have been studied (see e.g. Section 9.3 in [4] and [11, 26]).

Let $p$ be a prime and $q$ be a power of $p$. Let us denote by $\mathbb{F}_{q}$ the finite field of $q$ elements, $\mathbb{F}_{q}[T]$ the ring of all polynomials over $\mathbb{F}_{q}, \mathbb{F}_{q}(T)$ the field of all rational functions over $\mathbb{F}_{q}$, and $\mathbb{F}_{q}\left(\left(T^{-1}\right)\right)$ the field of all Laurent series over $\mathbb{F}_{q}$. For $\xi \in \mathbb{F}_{q}\left(\left(T^{-1}\right)\right) \backslash\{0\}$, we can write

$$
\xi=\sum_{n=N}^{\infty} a_{n} T^{-n},
$$

where $N \in \mathbb{Z}, a_{n} \in \mathbb{F}_{q}$ for all $n \geq N$, and $a_{N} \neq 0$. We define an absolute value on $\mathbb{F}_{q}\left(\left(T^{-1}\right)\right)$ by $|0|:=0$ and $|\xi|:=q^{-N}$. This absolute value can be uniquely extended to the algebraic closure of $\mathbb{F}_{q}\left(\left(T^{-1}\right)\right)$ and we continue to write $|\cdot|$ for the extended absolute value. We call an element of $\mathbb{F}_{q}\left(\left(T^{-1}\right)\right)$ an algebraic Laurent series if the element is algebraic over $\mathbb{F}_{q}(T)$. We can define Diophantine exponents $w_{n}$ and $w_{n}^{*}$ for Laurent series over a finite field in a similar way to the real case.

Mahler 21] proved that an analogue of the Roth Theorem in this framework does not hold, that is, there exists an algebraic Laurent series $\xi$ such that $w_{1}(\xi)>1$. Indeed, let $r$ be a power of $p$ and put $\xi:=\sum_{n=0}^{\infty} T^{-r^{n}}$. Then $\xi$ is an algebraic Laurent series of degree $r$ with $w_{1}(\xi)=r-1$. After that, several people investigated algebraic Laurent series for which the analogue of the Roth Theorem does not hold (see e.g. [15, 17, 23, 30, 33]). Furthermore, Thakur [34, 35] constructed explicit algebraic Laurent series for which the analogue of (11) for $n=r+1$ does not hold, where $r$ is a power of $p$. For example, for integers $m, n \geq 2$, he constructed algebraic Laurent series $\alpha_{m, n}$, with explicit equations and continued fractions, such that

$$
\operatorname{deg} \alpha_{m, n} \leq r^{m}+1, \quad \lim _{n \rightarrow \infty} E_{1}\left(\alpha_{m, n}\right)=2, \quad \lim _{n \rightarrow \infty} E_{r+1}\left(\alpha_{m, n}\right) \geq r^{m-1}+\frac{r-1}{(r+1) r} .
$$

Here, $E_{n}(\xi)$ measures the quality of approximations to $\xi$ by algebraic Laurent series of degree $n$ (see Section 2 for the precise definition and relation between $E_{n}$ and $w_{n}^{*}$ ). Since $w_{r+1}^{*}\left(\alpha_{m, n}\right)+1 \geq E_{r+1}\left(\alpha_{m, n}\right)$, we obtain

$$
\lim _{n \rightarrow \infty} w_{r+1}^{*}\left(\alpha_{m, n}\right) \geq r^{m-1}-\frac{r^{2}+1}{(r+1) r}
$$

which implies that an analogue of (10) does not hold for $\alpha_{m, n}$ with sufficiently large $m, n$. In this paper, we investigate the phenomenon that properties of the Diophantine exponents in characteristic zero are different from that of positive characteristic. More precisely, for an integer $n \geq 1$, we construct algebraic Laurent series $\xi$ such that $E_{1}(\xi)$ are large values and

$$
E_{1}(\xi)=\max \left\{E_{m}(\xi) \mid 1 \leq m \leq n\right\} .
$$

The author [25] showed that there exists $\xi \in \mathbb{F}_{q}\left(\left(T^{-1}\right)\right)$ for which $w_{2}(\xi)$ and $w_{2}^{*}(\xi)$ are different. In this paper, improving the method in [25], we solve the problem of whether or not there exists $\xi \in \mathbb{F}_{q}\left(\left(T^{-1}\right)\right)$ for which $w_{n}(\xi)$ and $w_{n}^{*}(\xi)$ are different for any $n \geq 3$.

This paper is organized as follows: We state the main results on the Diophantine exponents $w_{n}$ and $w_{n}^{*}$ for Laurent series over a finite field in Section 2, In Section 3, we prepare some lemmas in order to prove the main results. We collect the proofs of the main results in Section 4. It is well-known that finite automatons relate to algebraic Laurent series. In Section 5, we give properties of the Diophantine exponents for Laurent series 
whose coefficients are generated by finite automatons. We also give analogues of the main results for real and $p$-adic numbers.

\section{MAin RESUlts}

In this section, we state the main results about the Diophantine exponents for Laurent series over a finite field and give some problems associated to the main results.

We use the following notation throughout this paper. We denote by $\lfloor x\rfloor$ the integer part of a real number $x$. We use the Vinogradov notation $A \ll B$ if $|A| \leq c|B|$ for some constant $c>0$. We write $A \asymp B$ if $A \ll B$ and $B \ll A$. For a finite word $W$, we put $\bar{W}:=W W \cdots W \cdots$ (infinitely many concatenations of the word $W$ ). An infinite word $\mathbf{a}=a_{0} a_{1} \cdots$ is called ultimately periodic if there exist a finite word $U$ and a non-empty finite word $V$ such that $\mathbf{a}=U \bar{V}$. We identify a sequence $\left(a_{n}\right)_{n \geq 0}$ with the infinite word $a_{0} a_{1} \cdots a_{n} \cdots$.

We denote by $\left(\mathbb{F}_{q}[T]\right)[X]$ the set of all polynomials in $X$ over $\mathbb{F}_{q}[T]$. The height of a polynomial $P(X) \in\left(\mathbb{F}_{q}[T]\right)[X]$, denoted by $H(P)$, is defined to be the maximum of the absolute values of the coefficients of $P(X)$. We denote by $\left(\mathbb{F}_{q}[T]\right)[X]_{\min }$ the set of all nonconstant, irreducible, primitive polynomials in $\left(\mathbb{F}_{q}[T]\right)[X]$ whose leading coefficients are monic polynomials in $T$. For $\alpha \in \overline{\mathbb{F}_{q}(T)}$, there exists a unique $P(X) \in\left(\mathbb{F}_{q}[T]\right)[X]_{\text {min }}$ such that $P(\alpha)=0$. We call the polynomial $P(X)$ the minimal polynomial of $\alpha$. The height (resp. the degree, the inseparable degree) of $\alpha$, denoted by $H(\alpha)(\operatorname{resp} \cdot \operatorname{deg} \alpha$, insep $\alpha$ ), is defined to be the height of $P(X)$ (resp. the degree of $P(X)$, the inseparable degree of $P(X))$. We now define the Diophantine exponents for Laurent series over a finite field. Let $n \geq 1$ be an integer and $\xi$ be in $\mathbb{F}_{q}\left(\left(T^{-1}\right)\right)$. We denote by $w_{n}(\xi)$ (resp. $\left.w_{n}^{*}(\xi)\right)$ the supremum of the real numbers $w$ (resp. $w^{*}$ ) which satisfy

$$
0<|P(\xi)| \leq H(P)^{-w} \quad\left(\text { resp. } 0<|\xi-\alpha| \leq H(\alpha)^{-w^{*}-1}\right)
$$

for infinitely many $P(X) \in\left(\mathbb{F}_{q}[T]\right)[X]$ of degree at most $n$ (resp. $\alpha \in \overline{\mathbb{F}_{q}(T)}$ of degree at most $n)$. It is clear that $w_{1}(\xi)=w_{1}^{*}(\xi)$ for all $\xi \in \mathbb{F}_{q}\left(\left(T^{-1}\right)\right)$.

Let $n \geq 1$ be an integer and let $\xi \in \mathbb{F}_{q}\left(\left(T^{-1}\right)\right)$ be a Laurent series which is not algebraic of degree at most $n$. We denote by $E_{n}(\xi)$ the supremum of the real numbers $w$ which satisfy

$$
0<|\xi-\alpha| \leq H(\alpha)^{-w}
$$

for infinitely many algebraic Laurent series $\alpha \in \mathbb{F}_{q}\left(\left(T^{-1}\right)\right)$ of degree $n$. It is obvious that

$$
E_{1}(\xi)=w_{1}(\xi)+1=w_{1}^{*}(\xi)+1
$$

for all irrational Laurent series $\xi \in \mathbb{F}_{q}\left(\left(T^{-1}\right)\right)$. It is also obvious that

$$
w_{n}^{*}(\xi)+1 \geq \max \left\{E_{m}(\xi) \mid 1 \leq m \leq n\right\}
$$

for all $\xi \in \mathbb{F}_{q}\left(\left(T^{-1}\right)\right)$ which are not algebraic of degree at most $n$.

As in the classical continued fraction theory of real numbers, if $\xi \in \mathbb{F}_{q}\left(\left(T^{-1}\right)\right)$, then we can write

$$
\xi=a_{0}+\frac{1}{a_{1}+\frac{1}{a_{2}+\frac{1}{\cdots}}},
$$

where $a_{0}, a_{n} \in \mathbb{F}_{q}[T]$ and $\operatorname{deg} a_{n} \geq 1$ for all $n \geq 1$. We write $\xi=\left[a_{0}, a_{1}, a_{2}, \ldots\right]$. We call $a_{0}$ and $a_{n}$ the partial quotients of $\xi$.

Schmidt [30] and Thakur [33] studied the Diophantine exponent $w_{1}$ for algebraic Laurent series. Schmidt [30] introduced a classification of algebraic Laurent series as follows: Let 
$\alpha$ be in $\mathbb{F}_{q}\left(\left(T^{-1}\right)\right) \backslash \mathbb{F}_{q}(T)$. We say that $\alpha$ is of Class $I$ (resp. Class IA) if there exist an integer $s \geq 0$ and $R, S, T, U \in \mathbb{F}_{q}[T]$ with $R U-S T \neq 0$ (resp. $R U-S T \in \mathbb{F}_{q}^{\times}$) such that

$$
\alpha=\frac{R \alpha^{p^{s}}+S}{T \alpha^{p^{s}}+U} .
$$

For example, any quadratic Laurent series is of Class IA. Mathan 23] proved that the value of $w_{1}$ for a Laurent series of Class I is rational. However, it is not known whether or not there exists an algebraic Laurent series for which the value of $w_{1}$ is irrational. Let $r$ be a power of $p$. Schmidt [30] and Thakur [33] independently proved that for any rational number $1<w \leq r$, there exists an algebraic Laurent series $\xi \in \mathbb{F}_{q}\left(\left(T^{-1}\right)\right)$ of degree at most $r+1$ such that $w_{1}(\xi)=w$. It is well-known that $w_{1}(\xi)=1$ and $w_{1}(\eta) \geq 1$ hold for any quadratic Laurent series $\xi \in \mathbb{F}_{q}\left(\left(T^{-1}\right)\right)$ and irrational Laurent series $\eta \in \mathbb{F}_{q}\left(\left(T^{-1}\right)\right)$ (see e.g. Theorem 5.1 in [25] and Lemma 3.18). Therefore, we deduce that the set of all values taken by $w_{1}$ over the set of all Laurent series of Class IA is equal to the set of all rational numbers greater than or equal to 1. Chen [15] refined Schmidt and Thakur's result by showing that the degree of $\xi$ can be taken to be equal to $r+1$.

We partially extend Chen's result to $w_{n}$ and $w_{n}^{*}$ for $n \geq 2$.

Theorem 2.1. Let $d \geq 1$ be an integer and $w>2 d-1$ be a rational number. Then there exist a strictly increasing sequence of positive integers $\left(k_{j}\right)_{j \geq 1}$ and a sequence $\left(\xi_{j}\right)_{j \geq 1}$ such that, for any $j \geq 1, \xi_{j}$ is of Class IA of degree $p^{k_{j}}+1$, and

$$
w_{1}\left(\xi_{j}\right)=w_{1}^{*}\left(\xi_{j}\right)=\ldots=w_{d}\left(\xi_{j}\right)=w_{d}^{*}\left(\xi_{j}\right)=w .
$$

Remark. (i). By Lemma 3.18, we obtain $w \leq p^{k_{1}}$.

(ii). When $w=p^{s}$, where $s \geq 1$ is an integer, it is known that there exist Laurent series of Class IA which satisfy (44). Indeed, let $a \in \mathbb{F}_{q}[T]$ be a non-constant polynomial. We define a Laurent series $\xi_{a}$ of Class IA by

$$
\xi_{a}=\left[a, a^{p^{s}}, a^{p^{2 s}}, \ldots\right] .
$$

Then it is known that $\xi_{a}$ is algebraic of degree $p^{s}+1$ and satisfies $w_{1}\left(\xi_{a}\right)=p^{s}$ (see Theorem 1 (1) and Remarks (1) in [33]). Therefore, it follows from Lemma 3.18 that

$$
w_{n}\left(\xi_{a}\right)=w_{n}^{*}\left(\xi_{a}\right)=p^{s}
$$

for all $n \geq 1$.

(iii). By (2) and (3), we deduce that

$$
E_{1}\left(\xi_{j}\right)=\max \left\{E_{n}\left(\xi_{j}\right) \mid 1 \leq n \leq d\right\}=w+1,
$$

where the $\xi_{j}$ 's are algebraic Laurent series as in Theorem 2.1.

It is known that the values of $w_{1}$ can be determined through the partial quotients of continued fraction. In this paper, we extend this result to $w_{n}$ and $w_{n}^{*}$ for all $n \geq 1$ for a certain class of Laurent series. This is the key point of the proof of Theorem 2.1,

As mentioned in Section 1, it is known that $w_{n}(\xi)=w_{n}^{*}(\xi)$ for all real algebraic numbers $\xi$ and integers $n \geq 1$. The proof of this result depends on the Schmidt Subspace Theorem which is a generalization of the Roth Theorem. However, analogues of these theorems in positive characteristic do not hold (see Section 11). Therefore, we address the following problem.

Problem 2.2. Is it true that

$$
w_{n}(\xi)=w_{n}^{*}(\xi)
$$

for an integer $n \geq 1$ and an algebraic Laurent series $\xi$ ? 
Note that Theorem 2.1 gives a partial answer to Problem 2.2. If we remove the condition that $\xi$ is algebraic, then the answer to Problem 2.2 is not true (see Theorems 2.8 and 2.9 below).

We state some corollaries of Theorem 2.1

Corollary 2.3. Let $n \geq 1$ be an integer. Then the set of all values taken by $w_{n}$ (resp. $w_{n}^{*}$ ) over the set of all Laurent series of Class IA contains the set of all rational numbers greater than $2 n-1$.

We address the following natural problem arising from Corollary 2.3 .

Problem 2.4. Let $n \geq 1$ be an integer. Determine the set of all values taken by $w_{n}$ (resp. $w_{n}^{*}$ ) over the set of all algebraic Laurent series.

Since the sequence of degrees of $\xi_{j}$ tends to infinity under the conditions of Theorem 2.1, we obtain the following corollary.

Corollary 2.5. Let $d \geq 1$ be an integer and $w>2 d-1$ be a rational number. Then there exists a set $\left\{\xi_{j} \mid j \geq 1\right\}$ of linearly independent Laurent series of Class IA such that, for any $j \geq 1$

$$
w_{1}\left(\xi_{j}\right)=w_{1}^{*}\left(\xi_{j}\right)=\ldots=w_{d}\left(\xi_{j}\right)=w_{d}^{*}\left(\xi_{j}\right)=w .
$$

We obtain the following theorem in a similar method to the proof of Theorem 2.1.

Theorem 2.6. Let $d \geq 1$ be an integer and $w \geq 2 d-1$ be a real number. Then there exist uncountably many $\xi \in \mathbb{F}_{q}\left(\left(T^{-1}\right)\right)$ such that

$$
w_{1}(\xi)=w_{1}^{*}(\xi)=\ldots=w_{d}(\xi)=w_{d}^{*}(\xi)=w .
$$

Analogues of Theorem 2.6 for real and $p$-adic numbers are already given in 17, 9 .

For $\xi \in \mathbb{F}_{q}\left(\left(T^{-1}\right)\right)$, it is easily seen that $\left(w_{n}(\xi)\right)_{n \geq 1}$ and $\left(w_{n}^{*}(\xi)\right)_{n \geq 1}$ are increasing sequences with $0 \leq w_{n}(\xi), w_{n}^{*}(\xi) \leq+\infty$ for all $n \geq 1$. Therefore, we have $w_{n}(\xi)=$ $w_{n}^{*}(\xi)=0$ and $w_{n}(\eta)=w_{n}^{*}(\eta)=1$ for all $n \geq 1, \xi \in \mathbb{F}_{q}(T)$, and quadratic Laurent series $\eta \in \mathbb{F}_{q}\left(\left(T^{-1}\right)\right)$ by Theorem 5.1 in 25 and Lemma 3.18. It is immediate that for any $n \geq 1, w_{n}(\xi)=w_{n}^{*}(\xi)=+\infty$, where $\xi=\sum_{m=1}^{\infty} T^{-m !}$. Hence, we have the following corollary of Theorem 2.6 .

Corollary 2.7. For an integer $n \geq 1$, the set of all values taken by $w_{n}$ (resp. $\left.w_{n}^{*}\right)$ contains the set $\{0,1\} \cup[2 n-1,+\infty]$. Furthermore, the set of all values taken by $w_{1}$ (resp. $\left.w_{1}^{*}\right)$ is equal to $\{0\} \cup[1,+\infty]$.

We extend Theorems 1.1 and 1.2 in [25].

Theorem 2.8. Let $d \geq 2$ be an integer and $w \geq\left(3 d+2+\sqrt{9 d^{2}+4 d+4}\right) / 2$ be a real number. Let $a, b \in \mathbb{F}_{q}[T]$ be distinct non-constant polynomials. Let $\left(a_{n, w}\right)_{n \geq 1}$ be the sequence given by

$$
a_{n, w}= \begin{cases}b & \text { if } n=\left\lfloor w^{i}\right\rfloor \text { for some integer } i \geq 0, \\ a & \text { otherwise }\end{cases}
$$

Set $\xi_{w}:=\left[0, a_{1, w}, a_{2, w}, \ldots\right]$. Then we have

$$
w_{n}^{*}\left(\xi_{w}\right)=w-1, \quad w_{n}\left(\xi_{w}\right)=w
$$

for all $2 \leq n \leq d$. 
Theorem 2.9. Let $d \geq 2$ be an integer, $w \geq 121 d^{2}$ be a real number, and $a, b, c \in \mathbb{F}_{q}[T]$ be distinct non-constant polynomials. Let $0<\eta<\sqrt{w} / d$ be a positive number and put $m_{i}:=\left\lfloor\left(\left\lfloor w^{i+1}\right\rfloor-\left\lfloor w^{i}-1\right\rfloor\right) /\left\lfloor\eta w^{i}\right\rfloor\right\rfloor$ for all $i \geq 1$. Let $\left(a_{n, w, \eta}\right)_{n \geq 1}$ be the sequence given by

$$
a_{n, w, \eta}= \begin{cases}b & \text { if } n=\left\lfloor w^{i}\right\rfloor \text { for some integer } i \geq 0, \\ c \quad \text { if } n \neq\left\lfloor w^{i}\right\rfloor \text { for all integers } i \geq 0 \text { and } n=\left\lfloor w^{j}\right\rfloor+ & \\ & m\left\lfloor\eta w^{j}\right\rfloor \text { for some integers } 1 \leq m \leq m_{j}, j \geq 1, \\ a & \text { otherwise. }\end{cases}
$$

Set $\xi_{w, \eta}:=\left[0, a_{1, w, \eta}, a_{2, w, \eta}, \ldots\right]$. Then we have

$$
w_{n}^{*}\left(\xi_{w, \eta}\right)=\frac{2 w-2-\eta}{2+\eta}, \quad w_{n}\left(\xi_{w, \eta}\right)=\frac{2 w-\eta}{2+\eta}
$$

for all $2 \leq n \leq d$. Hence, we have

$$
w_{n}\left(\xi_{w, \eta}\right)-w_{n}^{*}\left(\xi_{w, \eta}\right)=\frac{2}{2+\eta}
$$

for all $2 \leq n \leq d$.

Theorems 2.8 and 2.9 imply that for each $n \geq 2$, we can construct explicitly Laurent series $\xi$ for which $w_{n}(\xi)$ and $w_{n}^{*}(\xi)$ are different. The general strategies of the proof of Theorems 2.8 and 2.9 are same as that of Theorems 1.1 and 1.2 in 25]. The key ingredient is that for $n \geq 3$, if $\xi \in \mathbb{F}_{q}\left(\left(T^{-1}\right)\right)$ has a dense (in a suitable sense) sequence of very good quadratic approximations, then we can determine $w_{n}(\xi)$ and $w_{n}^{*}(\xi)$.

The following corollary is immediate from Theorems 2.6, 2.8, and 2.9.

Corollary 2.10. Let $d \geq 2$ be an integer and $\delta$ be in the closed interval $[0,1]$. Then there exist uncountably many $\xi \in \mathbb{F}_{q}\left(\left(T^{-1}\right)\right)$ such that $w_{n}(\xi)-w_{n}^{*}(\xi)=\delta$ for all $2 \leq n \leq d$. In particular, the set of all values taken by $w_{d}-w_{d}^{*}$ contains the closed interval $[0,1]$.

Note that it is already known that the set of all values taken by $w_{2}-w_{2}^{*}$ is the closed interval $[0,1]$ in $[25$. 2.10

In the last part of this section, we mention a problem associated to Corollary 2.7 and

Problem 2.11. Let $n \geq 1$ be an integer. Determine the set of all values taken by $w_{n}$ $\left(\right.$ resp. $\left.w_{n}^{*}, w_{n}-w_{n}^{*}\right)$.

\section{Preliminaries}

Let $\xi$ be in $\mathbb{F}_{q}\left(\left(T^{-1}\right)\right)$ and $n \geq 1$ be an integer. We denote by $\tilde{w}_{n}(\xi)$ the supremum of the real numbers $w$ which satisfy

$$
0<|P(\xi)| \leq H(P)^{-w}
$$

for infinitely many $P(X) \in\left(\mathbb{F}_{q}[T]\right)[X]_{\text {min }}$ of degree at most $n$.

Lemma 3.1. Let $n \geq 1$ be an integer and $\xi$ be in $\mathbb{F}_{q}\left(\left(T^{-1}\right)\right)$. Then we have

$$
w_{n}(\xi)=\tilde{w}_{n}(\xi) \text {. }
$$

Proof. See Lemma 5.3 in [25].

Lemma 3.2. Let $\alpha, \beta$ be in $\mathbb{F}_{q}\left(\left(T^{-1}\right)\right)$ and $P(X) \in\left(\mathbb{F}_{q}[T]\right)[X]$ be a non-constant polynomial of degree $d$. Let $C \geq 0$ be a real number. Assume that $|\alpha-\beta| \leq C$. Then there exists a positive constant $C_{1}(\alpha, C, d)$, depending only on $\alpha, C$, and $d$, such that

$$
|P(\alpha)-P(\beta)| \leq C_{1}(\alpha, C, d)|\alpha-\beta| H(P) .
$$


Proof. It is easily seen that

$$
|P(\alpha)-P(\beta)| \leq H(P) \max _{1 \leq i \leq d}\left|\alpha^{i}-\beta^{i}\right| .
$$

By the assumption, we have $\max (C,|\alpha|)=\max (C,|\beta|)$. For any $1 \leq i \leq d$, we obtain

$$
\begin{aligned}
\left|\alpha^{i}-\beta^{i}\right| & =|\alpha-\beta|\left|\sum_{j=0}^{i-1} \alpha^{j} \beta^{i-1-j}\right| \leq|\alpha-\beta| \max _{0 \leq j \leq i-1}\left|\alpha^{j} \beta^{i-1-j}\right| \\
& \leq|\alpha-\beta| \max _{1 \leq k \leq d}(C,|\alpha|)^{k-1} .
\end{aligned}
$$

Hence, we have (7).

Lemma 3.3. Let $n \geq 1$ be an integer and $\xi$ be in $\mathbb{F}_{q}\left(\left(T^{-1}\right)\right)$. Then we have

$$
w_{n}^{*}(\xi) \leq w_{n}(\xi)
$$

Proof. See Proposition 5.6 in [25].

Lemma 3.4. Let $\xi$ be in $\mathbb{F}_{q}\left(\left(T^{-1}\right)\right), d \geq 1$ be an integer, and $\theta, \rho, \delta$ be positive numbers. Assume that there exists a sequence $\left(p_{j} / q_{j}\right)_{j \geq 1}$ with $p_{j}, q_{j} \in \mathbb{F}_{q}[T], q_{j} \neq 0, \operatorname{gcd}\left(p_{j}, q_{j}\right)=1$ for any $j \geq 1$ such that $\left(\left|q_{j}\right|\right)_{j \geq 1}$ is a divergent increasing sequence, and

$$
\begin{gathered}
\limsup _{j \rightarrow \infty} \frac{\log \left|q_{j+1}\right|}{\log \left|q_{j}\right|} \leq \theta, \\
d+\delta \leq \liminf _{j \rightarrow \infty} \frac{-\log \left|\xi-p_{j} / q_{j}\right|}{\log \left|q_{j}\right|}, \quad \limsup _{j \rightarrow \infty} \frac{-\log \left|\xi-p_{j} / q_{j}\right|}{\log \left|q_{j}\right|} \leq d+\rho .
\end{gathered}
$$

Then we have for all $1 \leq n \leq d$

$$
d-1+\delta \leq w_{n}^{*}(\xi) \leq w_{n}(\xi) \leq \max \left(d-1+\rho, \frac{d \theta}{\delta}\right) .
$$

Note that Lemma 3.4 is a generalization of analogue of Lemma 1 in [2].

Proof. Let $0<\iota<\delta$ be a real number. By the assumption, there exists an integer $c_{0} \geq 1$ such that

$$
\left|q_{j}\right| \leq\left|q_{j+1}\right| \leq\left|q_{j}\right|^{\theta+\iota}, \quad \frac{1}{\left|q_{j}\right|^{d+\rho+\iota}} \leq\left|\xi-\frac{p_{j}}{q_{j}}\right| \leq \frac{1}{\left|q_{j}\right|^{d+\delta-\iota}}
$$

for all $j \geq c_{0}$. Since $\left|\xi-p_{j} / q_{j}\right| \leq 1$ for $j \geq c_{0}$, we have $\left|q_{j}\right| \max (1,|\xi|)=\max \left(\left|p_{j}\right|,\left|q_{j}\right|\right)=$ $H\left(p_{j} / q_{j}\right)$ for $j \geq c_{0}$. Therefore, we obtain

$$
0<\left|\xi-\frac{p_{j}}{q_{j}}\right| \leq \frac{\max (1,|\xi|)^{d+\delta}}{H\left(p_{j} / q_{j}\right)^{d+\delta-\iota}}
$$

for $j \geq c_{0}$. Since $\iota$ is arbitrary, we have

$$
d-1+\delta \leq w_{1}^{*}(\xi) \leq w_{2}^{*}(\xi) \leq \ldots \leq w_{d}^{*}(\xi) .
$$

By Lemma 3.3, it is sufficient to show that

$$
w_{d}(\xi) \leq \max \left(d-1+\rho, \frac{d \theta}{\delta}\right) .
$$

Put $c_{1}:=\max (1,|\xi|)^{d-1}$. Let $P(X) \in\left(\mathbb{F}_{q}[T]\right)[X]_{\min }$ be a polynomial of degree at most $d$ with $H(P) \geq c_{1}^{-1}\left|q_{c_{0}}\right|^{\frac{\delta}{\theta}}$. We first consider the case where $P\left(p_{j} / q_{j}\right)=0$ for some $j \geq c_{0}$. Then we can write $P(X)=a_{j}\left(q_{j} X-p_{j}\right)$ for some $a_{j} \in \mathbb{F}_{q}$. Therefore, we have

$$
|P(\xi)| \geq\left|q_{j}\right|^{-d+1-\rho-\iota} \geq H(P)^{-d+1-\rho-\iota} .
$$


We now turn to the case where $P\left(q_{j} / p_{j}\right) \neq 0$ for all $j \geq c_{0}$. We define an integer $j_{0} \geq c_{0}$ by $\left|q_{j_{0}}\right| \leq\left(c_{1} H(P)\right)^{\frac{\theta+\iota}{\delta-\iota}}<\left|q_{j_{0}+1}\right|$. Then we have

$$
H(P)<c_{1}^{-1}\left|q_{j_{0}+1}\right|^{\frac{\delta-\iota}{\theta+\iota}} \leq c_{1}^{-1}\left|q_{j_{0}}\right|^{\delta-\iota} .
$$

It follows from Lemma 3.2 that

$$
\left|P(\xi)-P\left(p_{j_{0}} / q_{j_{0}}\right)\right| \leq c_{1} H(P)\left|\xi-\frac{p_{j_{0}}}{q_{j_{0}}}\right|<\left|q_{j_{0}}\right|^{-d} .
$$

Since $\left|P\left(p_{j_{0}} / q_{j_{0}}\right)\right| \geq\left|q_{j_{0}}\right|^{-d}$, we obtain

$$
|P(\xi)|=\left|P\left(p_{j_{0}} / q_{j_{0}}\right)\right| \geq\left|q_{j_{0}}\right|^{-d} \geq\left(c_{1} H(P)\right)^{-\frac{d(\theta+\iota)}{\delta-\iota}} .
$$

Therefore, by Lemma 3.1, we have

$$
w_{d}(\xi) \leq \max \left(d-1+\rho+\iota, \frac{d(\theta+\iota)}{\delta-\iota}\right) .
$$

Since $\iota$ is arbitrary, we obtain (9).

Let $\xi$ be in $\mathbb{F}_{q}\left(\left(T^{-1}\right)\right)$ and we denote by $\left[a_{0}, a_{1}, a_{2}, \ldots\right]$ the continued fraction expansion of $\xi$. We define sequences $\left(p_{n}\right)_{n \geq-1}$ and $\left(q_{n}\right)_{n \geq-1}$ by

$$
\left\{\begin{array}{l}
p_{-1}=1, p_{0}=a_{0}, p_{n}=a_{n} p_{n-1}+p_{n-2}, n \geq 1, \\
q_{-1}=0, q_{0}=1, q_{n}=a_{n} q_{n-1}+q_{n-2}, n \geq 1 .
\end{array}\right.
$$

We call $\left(p_{n} / q_{n}\right)_{n \geq 0}$ the convergent sequence of $\xi$. We gather fundamental properties of continued fractions in the following lemma.

Lemma 3.5. Let $\xi=\left[a_{0}, a_{1}, a_{2}, \ldots\right]$ be in $\mathbb{F}_{q}\left(\left(T^{-1}\right)\right)$ and $\left(p_{n} / q_{n}\right)_{n \geq 0}$ be the convergent sequence of $\xi$. Then the following hold:

(i) $\frac{p_{n}}{q_{n}}=\left[a_{0}, a_{1}, \ldots a_{n}\right] \quad(n \geq 0)$,

(ii) $\operatorname{gcd}\left(p_{n}, q_{n}\right)=1 \quad(n \geq 0)$,

(iii) $\left|q_{n}\right|=\left|a_{1}\right|\left|a_{2}\right| \cdots\left|a_{n}\right| \quad(n \geq 1)$

(iv) $\left|\xi-\frac{p_{n}}{q_{n}}\right|=\frac{1}{\left|q_{n}\right|\left|q_{n+1}\right|}=\frac{1}{\left|a_{n+1}\right|\left|q_{n}\right|^{2}} \quad(n \geq 0)$,

(v) $\xi=\frac{\xi_{n+1} p_{n}+p_{n-1}}{\xi_{n+1} q_{n}+q_{n-1}}$, where $\xi_{n+1}=\left[a_{n+1}, a_{n+2}, \ldots\right] \quad(n \geq 0)$.

The following lemma is a well-known result (see e.g. [19, 34]).

Lemma 3.6. Let $\xi=\left[a_{0}, a_{1}, a_{2}, \ldots\right]$ be in $\mathbb{F}_{q}\left(\left(T^{-1}\right)\right)$ and $\left(p_{n} / q_{n}\right)_{n \geq 0}$ be the convergent sequence of $\xi$. Then we have

$$
w_{1}(\xi)=w_{1}^{*}(\xi)=\limsup _{n \rightarrow \infty} \frac{\operatorname{deg} q_{n+1}}{\operatorname{deg} q_{n}} .
$$

We extend Lemma 3.6 by using Lemma 3.4.

Proposition 3.7. Let $d \geq 1$ be an integer and $\xi=\left[a_{0}, a_{1}, a_{2}, \ldots\right]$ be in $\mathbb{F}_{q}\left(\left(T^{-1}\right)\right)$. Let $\left(p_{n} / q_{n}\right)_{n \geq 0}$ be the convergent sequence of $\xi$. Assume that

$$
\liminf _{n \rightarrow \infty} \frac{\operatorname{deg} q_{n+1}}{\operatorname{deg} q_{n}} \geq 2 d-1
$$

Then we have

$$
w_{1}(\xi)=w_{1}^{*}(\xi)=\ldots=w_{d}(\xi)=w_{d}^{*}(\xi)=\limsup _{n \rightarrow \infty} \frac{\operatorname{deg} q_{n+1}}{\operatorname{deg} q_{n}} .
$$


Proof. For $j \geq 1$, put

$$
A_{j}:=\frac{\operatorname{deg} q_{j+1}}{\operatorname{deg} q_{j}}=\frac{\log \left|q_{j+1}\right|}{\log \left|q_{j}\right|} .
$$

It follows from Lemmas 3.3 and 3.6 that for all $n \geq 1$,

$$
\limsup _{j \rightarrow \infty} A_{j} \leq w_{n}^{*}(\xi) \leq w_{n}(\xi)
$$

By Lemma 3.5 (iv), we have

$$
\frac{-\log \left|\xi-p_{j} / q_{j}\right|}{\log \left|q_{j}\right|}=1+A_{j}
$$

for all $j \geq 1$. It follows from Lemma 3.5 (iii) and (iii) that $q_{j} \neq 0$ and $\operatorname{gcd}\left(p_{j}, q_{j}\right)=1$ for all $j \geq 1$. Moreover, the positive integer sequence $\left(\left|q_{j}\right|\right)_{j \geq 1}$ is strictly increasing, which implies that it is divergent. Applying Lemma 3.4 with

$$
\theta=\limsup _{j \rightarrow \infty} A_{j}, \quad \delta=d, \quad \rho=1+\limsup _{j \rightarrow \infty} A_{j}-d,
$$

we obtain

$$
w_{n}^{*}(\xi) \leq w_{n}(\xi) \leq \limsup _{j \rightarrow \infty} A_{j}
$$

for all $1 \leq n \leq d$. Hence, we have (10).

Schmidt [30] characterized Laurent series of Class IA by using continued fractions.

Theorem 3.8. Let $\alpha$ be in $\mathbb{F}_{q}\left(\left(T^{-1}\right)\right)$. Then $\alpha$ is of Class IA if and only if the continued fraction expansion of $\alpha$ is of the form

$$
\alpha=\left[a_{1}, a_{2}, \ldots, a_{t}, b_{1}, b_{2}, \ldots\right],
$$

where $t \geq 0$ and

$$
b_{j+s}= \begin{cases}a b_{j}^{p^{k}} & \text { when } j \text { is odd, } \\ a^{-1} b_{j}^{p^{k}} & \text { when } j \text { is even }\end{cases}
$$

for some $a \in \mathbb{F}_{q}^{\times}$, integers $s \geq 1$ and $k \geq 0$.

Proof. See Theorem 4 in [30].

Thakur [33] worked at the ratios of the degrees of the denominators of the convergent sequences for Laurent series of Class IA.

Theorem 3.9. Let $\alpha \in \mathbb{F}_{q}\left(\left(T^{-1}\right)\right)$ be as in (11) and (12), and $\left(p_{n} / q_{n}\right)_{n \geq 0}$ be the convergent sequence of $\alpha$. Put $d_{i}:=\operatorname{deg} b_{i}$ and

$$
r_{i}:=\frac{d_{i}}{p^{k}\left(\sum_{j=1}^{i-1} d_{j}\right)+\sum_{j=i}^{s} d_{j}} .
$$

Then we have

$$
\begin{aligned}
& \limsup _{n \rightarrow \infty} \frac{\operatorname{deg} q_{n+1}}{\operatorname{deg} q_{n}}=1+\left(p^{k}-1\right) \max \left\{r_{1}, \ldots, r_{s}\right\}, \\
& \liminf _{n \rightarrow \infty} \frac{\operatorname{deg} q_{n+1}}{\operatorname{deg} q_{n}}=1+\left(p^{k}-1\right) \min \left\{r_{1}, \ldots, r_{s}\right\} .
\end{aligned}
$$

Proof. We have (13) by Theorem 1 (1) in 33, and Lemma 3.6, Meanwhile, (14) follows in a similar way to the proof of Theorem 1 (1) in 33 .

We define a valuation $v$ on $\mathbb{F}_{q}\left(\left(T^{-1}\right)\right)$ by $v(\xi)=-\log _{q}|\xi|$ for $\xi \in \mathbb{F}_{q}\left(\left(T^{-1}\right)\right)$. 
Lemma 3.10. Let

$$
P(X)=X^{m}+\sum_{i=1}^{m} a_{i} X^{m-i}
$$

be a monic polynomial in $\left(\mathbb{F}_{q}\left(\left(T^{-1}\right)\right)\right)[X]$. Assume that $v\left(a_{m}\right)$ is a positive integer with $\operatorname{gcd}\left(v\left(a_{m}\right), m\right)=1$. Then $P(X)$ is irreducible over $\mathbb{F}_{q}\left(\left(T^{-1}\right)\right)$ if and only if $v\left(a_{i}\right) / i>$ $v\left(a_{m}\right) / m$ for all $1 \leq i \leq m-1$.

Proof. See Proposition 2.2 in [27].

We give a sufficient condition to determine degrees of Laurent series of Class IA. The following lemma is inspired by the proof of Theorem 2 in [15].

Lemma 3.11. Let $\alpha \in \mathbb{F}_{q}\left(\left(T^{-1}\right)\right)$ be as in (11) and (12). If $\operatorname{gcd}\left(\operatorname{deg} b_{s}, p\right)=1$, then $\alpha$ is algebraic of degree $p^{k}+1$.

Proof. By Lemma 3.5 (四), it is sufficient to show that $\beta=\left[b_{1}, b_{2}, \ldots\right]$ is algebraic of degree $p^{k}+1$. Let $\left(p_{n} / q_{n}\right)_{n \geq 0}$ be the convergent sequence of $\beta$. Since

$$
\beta_{s}=\left[a b_{1}^{p^{k}}, a^{-1} b_{2}^{p^{k}}, \ldots\right]=a \beta^{p^{k}},
$$

it follows from Lemma 3.5 (v) that $\beta$ is a root of the monic polynomial

$$
P(X):=X^{p^{k}+1}-\frac{p_{s-1}}{q_{s-1}} X^{p^{k}}+\frac{q_{s-2}}{a q_{s-1}} X-\frac{p_{s-2}}{a q_{s-1}} .
$$

Put

$$
Q(X):=X^{p^{k}}+\left(\beta-\frac{p_{s-1}}{q_{s-1}}\right) \sum_{j=0}^{p^{k}-1} \beta^{p^{k}-1-j} X^{j}+\frac{q_{s-2}}{a q_{s-1}},
$$

then we have $P(X)=(X-\beta) Q(X)$. For $1 \leq i \leq p^{k}$, let $c_{i}$ be the coefficient of $X^{p^{k}-i}$ in $Q(X)$. By Lemma 3.5 (iii) and (iv), we deduce that

$$
\begin{gathered}
v\left(c_{i}\right)=\left(p^{k}-i+1\right) \operatorname{deg} b_{1}+2 \sum_{j=2}^{s} \operatorname{deg} b_{j} \quad\left(1 \leq i \leq p^{k}-1\right), \\
v\left(c_{p^{k}}\right)=\operatorname{deg} b_{s} .
\end{gathered}
$$

Therefore, by Lemma 3.10, the monic polynomial $Q(X)$ is irreducible over $\mathbb{F}_{q}\left(\left(T^{-1}\right)\right)$. Since $\beta \notin \mathbb{F}_{q}(T)$, we derive that $P(X)$ is irreducible over $\mathbb{F}_{q}(T)$.

Lemma 3.12. Let $d \geq 1$ be an integer and $w>2 d-1$ be a rational number. Write $w=a / b$, where $a, b \geq 1$ are integers and $a=p^{m} a^{\prime}$, where $a^{\prime} \geq 1, m \geq 0$ are integers and $\operatorname{gcd}\left(a^{\prime}, p\right)=1$. Then there exist a strictly increasing sequence of positive integers $\left(k_{j}\right)_{j \geq 1}$, a sequence of integers $\left(n_{j}\right)_{j \geq 1}$, and a sequence of rational numbers $\left(u_{j}\right)_{j \geq 1}$ such that for any $j \geq 1, n_{j} \geq 3, u_{j}=a_{j} / b_{j}$, where $a_{j}, b_{j} \geq 1$ are integers, $\operatorname{gcd}\left(a_{j}, p\right)=1, p^{m} \mid b_{j}$, and

$$
2 d-1<\min \left\{w, u_{j}, \frac{p^{k_{j}}}{w u_{j}^{n_{j}-2}}\right\}, \quad \max \left\{w, u_{j}, \frac{p^{k_{j}}}{w u_{j}^{n_{j}-2}}\right\}=w .
$$

Proof. The proof is by induction on $j$. By assumption, we take an integer $n_{1} \geq 3$ with $(w /(2 d-1))^{n_{1}-1}>p$. Then we have $\log _{p} w^{n_{1}}-\log _{p} w(2 d-1)^{n_{1}-1}>1$. This implies that there exists an integer $k_{1} \geq 1$ such that

$$
w(2 d-1)^{n_{1}-1}<p^{k_{1}}<w^{n_{1}} .
$$

Then we have

$$
\max \left\{2 d-1,\left(\frac{p^{k_{1}}}{w^{2}}\right)^{\frac{1}{n_{1}-2}}\right\}<\min \left\{w,\left(\frac{p^{k_{1}}}{(2 d-1) w}\right)^{\frac{1}{n_{1}-2}}\right\} .
$$


Let $r \geq 2$ be an integer such that $\operatorname{gcd}(r, p)=1$. By Lemma 2.5.9 in [1], the set $\left\{r^{y} / p^{x} \mid\right.$ $\left.x, y \in \mathbb{Z}_{\geq 0}\right\}$ is dense in $\mathbb{R}_{>0}$. Therefore, we can take a rational number $u_{1}=a_{1} / b_{1}$ such that $a_{1}, \bar{b}_{1} \in \mathbb{Z}_{>0}, \operatorname{gcd}\left(a_{1}, p\right)=1, p^{m} \mid b_{1}$, and

$$
\max \left\{2 d-1,\left(\frac{p^{k_{1}}}{w^{2}}\right)^{\frac{1}{n_{1}-2}}\right\}<u_{1}<\min \left\{w,\left(\frac{p^{k_{1}}}{(2 d-1) w}\right)^{\frac{1}{n_{1}-2}}\right\} .
$$

Thus, we have (15) when $j=1$. Assume that (15) holds for $j=1, \ldots, i$. We take an integer $n_{i+1} \geq 3$ with with $(w /(2 d-1))^{n_{i+1}-1}>p^{k_{i}}$. In a similar way to the above proof, we can take an integer $k_{i+1}$ with $k_{i}<k_{i+1}$ and a rational number $u_{i+1}$ which satisfy (15). This completes the proof.

Quadratic Laurent series are characterized by continued fractions as follows:

Lemma 3.13. Let $\xi=\left[a_{0}, a_{1}, \ldots\right]$ be in $\mathbb{F}_{q}\left(\left(T^{-1}\right)\right)$. Then $\xi$ is quadratic if and only if $\left(a_{n}\right)_{n \geq 0}$ is ultimately periodic.

Proof. See e.g. Théorème 4 in [22, CHAPITRE IV] or Theorems 3 and 4 in [14].

The following lemma is well-known and immediately seen.

Lemma 3.14. Let $P(X)$ be in $\left(\mathbb{F}_{q}[T]\right)[X]$. Assume that $P(X)$ can be factorized as

$$
P(X)=A \prod_{i=1}^{n}\left(X-\alpha_{i}\right)
$$

where $A \in \mathbb{F}_{q}[T]$ and $\alpha_{i} \in \overline{\mathbb{F}_{q}(T)}$ for $1 \leq i \leq n$. Then we have

$$
H(P)=|A| \prod_{i=1}^{n} \max \left(1,\left|\alpha_{i}\right|\right) .
$$

Furthermore, for $P(X), Q(X) \in\left(\mathbb{F}_{q}[T]\right)[X]$, we have

$$
H(P Q)=H(P) H(Q) \text {. }
$$

Let $\alpha \in \overline{\mathbb{F}_{q}(T)}$ be a quadratic number. If insep $\alpha=1$, let $\alpha^{\prime} \neq \alpha$ be the Galois conjugate of $\alpha$. If insep $\alpha=2$, let $\alpha^{\prime}=\alpha$.

Lemma 3.15. Let $\alpha \in \overline{\mathbb{F}_{q}(T)}$ be a quadratic number. If $\alpha \neq \alpha^{\prime}$, then we have

$$
\left|\alpha-\alpha^{\prime}\right| \geq H(\alpha)^{-1} \text {. }
$$

Proof. This is clear by using the discriminant of the minimal polynomial of $\alpha$ (see e.g. [13, Appendix A] for the definition and properties of the discriminant). We refer to Lemma 3.5 in [25] for a direct proof.

We recall the Liouville inequalities for Laurent series over a finite field.

Lemma 3.16. Let $P(X) \in\left(\mathbb{F}_{q}[T]\right)[X]$ be a non-constant polynomial of degree $m$ and $\alpha \in \overline{\mathbb{F}_{q}(T)}$ be a number of degree $n$. Assume that $P(\alpha) \neq 0$. Then we have

$$
|P(\alpha)| \geq H(P)^{-n+1} H(\alpha)^{-m} .
$$

Proof. See e.g. Lemma 4 in [24] or Proposition 3.2 in [25].

Lemma 3.17. Let $\alpha, \beta \in \overline{\mathbb{F}_{q}(T)}$ be distinct numbers of degree $m$ and $n$, respectively. Then we have

$$
|\alpha-\beta| \geq H(\alpha)^{-n} H(\beta)^{-m} .
$$

Proof. See e.g. Korollar 3 in [24] or Proposition 3.4 in [25]. 
The lemma below is an immediate consequence of Lemmas 3.16 and 3.17 .

Lemma 3.18. Let $n \geq 1$ be an integer and $\xi$ be an algebraic Laurent series of degree $d$. Then we have

$$
w_{n}(\xi), w_{n}^{*}(\xi) \leq d-1 .
$$

We give a key lemma for the proof of Theorems 2.8 and 2.9 as follows:

Lemma 3.19. Let $d \geq 2$ be an integer. Let $\xi$ be in $\mathbb{F}_{q}\left(\left(T^{-1}\right)\right)$ and $\theta, \rho, \delta$ be positive numbers. Assume that there exists a sequence $\left(\alpha_{j}\right)_{j \geq 1}$ such that for any $j \geq 1, \alpha_{j} \in \overline{\mathbb{F}_{q}(T)}$ is quadratic, $\left(H\left(\alpha_{j}\right)\right)_{j \geq 1}$ is a divergent increasing sequence, and

$$
\begin{gathered}
\limsup _{j \rightarrow \infty} \frac{\log H\left(\alpha_{j+1}\right)}{\log H\left(\alpha_{j}\right)} \leq \theta, \\
d+\delta \leq \liminf _{j \rightarrow \infty} \frac{-\log \left|\xi-\alpha_{j}\right|}{\log H\left(\alpha_{j}\right)}, \quad \limsup _{j \rightarrow \infty} \frac{-\log \left|\xi-\alpha_{j}\right|}{\log H\left(\alpha_{j}\right)} \leq d+\rho .
\end{gathered}
$$

If $2 d \theta \leq(d-2+\rho) \delta$, then we have for all $2 \leq n \leq d$,

$$
d-1+\delta \leq w_{n}^{*}(\xi) \leq d-1+\rho .
$$

Furthermore, assume that there exist a non-negative number $\varepsilon$ and a positive number $c$ such that for any $j \geq 1,0<\left|\alpha_{j}-\alpha_{j}^{\prime}\right| \leq c$ and

$$
\limsup _{j \rightarrow \infty} \frac{-\log \left|\alpha_{j}-\alpha_{j}^{\prime}\right|}{\log H\left(\alpha_{j}\right)} \geq \varepsilon
$$

If $2 d \theta \leq(d-2+\delta) \delta$, then we have for all $2 \leq n \leq d$,

$$
d-1+\delta \leq w_{n}^{*}(\xi) \leq d-1+\rho, \quad \varepsilon \leq w_{n}(\xi)-w_{n}^{*}(\xi) .
$$

Finally, assume that there exists a non-negative number $\chi$ such that

$$
\limsup _{i \rightarrow \infty} \frac{-\log \left|\alpha_{i}-\alpha_{i}^{\prime}\right|}{\log H\left(\alpha_{i}\right)} \leq \chi
$$

Then we have for all $2 \leq n \leq d$,

$$
d-1+\delta \leq w_{n}^{*}(\xi) \leq d-1+\rho, \quad \varepsilon \leq w_{n}(\xi)-w_{n}^{*}(\xi) \leq \chi .
$$

Proof. Let $0<\iota<\delta$ be a real number. Then there exists a positive integer $c_{0}$ such that

$$
\frac{1}{H\left(\alpha_{j}\right)^{d+\rho+\iota}} \leq\left|\xi-\alpha_{j}\right| \leq \frac{1}{H\left(\alpha_{j}\right)^{d+\delta-\iota}}, \quad H\left(\alpha_{j}\right) \leq H\left(\alpha_{j+1}\right) \leq H\left(\alpha_{j}\right)^{\theta+\iota}
$$

for all $j \geq c_{0}$. Since $\iota$ is arbitrary, we have $w_{2}^{*}(\xi) \geq d-1+\delta$. Let $\alpha \in \overline{\mathbb{F}_{q}(T)} \backslash\left\{\alpha_{j} \mid j \geq 1\right\}$ be an algebraic number of degree at most $d$ with $H(\alpha) \geq H\left(\alpha_{c_{0}}\right)^{\frac{\delta}{2 \theta}}$.

Assume that $2 d \theta \leq(d-2+\rho) \delta$. We define an integer $j_{0} \geq c_{0}$ such that $H\left(\alpha_{j_{0}}\right) \leq$ $H(\alpha)^{\frac{2(\theta+\iota)}{\delta-\iota}}<H\left(\alpha_{j_{0}+1}\right)$. Since

$$
H(\alpha)<H\left(\alpha_{j_{0}+1}\right)^{\frac{\delta-\iota}{2(\theta+\iota)}} \leq H\left(\alpha_{j_{0}}\right)^{\frac{\delta-\iota}{2}},
$$

we obtain

$$
\left|\alpha-\alpha_{j_{0}}\right| \geq H(\alpha)^{-2} H\left(\alpha_{j_{0}}\right)^{-d}>H\left(\alpha_{j_{0}}\right)^{-d-\delta+\iota} \geq\left|\xi-\alpha_{j_{0}}\right|
$$

by Lemma 3.17, We derive that

$$
|\xi-\alpha|=\left|\alpha-\alpha_{j_{0}}\right| \geq H(\alpha)^{-2} H\left(\alpha_{j_{0}}\right)^{-d} \geq H(\alpha)^{-2-\frac{2 d(\theta+\iota)}{\delta-\iota}},
$$

which implies

$$
w_{d}^{*}(\xi) \leq \max \left(d-1+\rho+\iota, 1+\frac{2 d(\theta+\iota)}{\delta-\iota}\right)
$$


Since $\iota$ is arbitrarily small, (18) follows.

Next, we assume that $2 d \theta \leq(d-2+\delta) \delta$ and there exist a non-negative number $\varepsilon$ and a positive number $c$ such that for any $j \geq 1,0<\left|\alpha_{j}-\alpha_{j}^{\prime}\right| \leq c$ and (19) holds. Since $\delta \leq \rho$, we have (18). By the assumption and (23), the sequence $\left(\alpha_{j}\right)_{j \geq 1}$ is the best approximation to $\xi$ of degree at most $d$, that is,

$$
w_{d}^{*}(\xi)=\limsup _{j \rightarrow \infty} \frac{-\log \left|\xi-\alpha_{j}\right|}{\log H\left(\alpha_{j}\right)}-1 .
$$

Therefore, we have $w_{2}^{*}(\xi)=\ldots=w_{d}^{*}(\xi)$. In what follows, we show that $\varepsilon \leq w_{n}(\xi)-w_{n}^{*}(\xi)$ for all $2 \leq n \leq d$. For any $j \geq 1$, we denote by $P_{j}(X)=A_{j}\left(X-\alpha_{j}\right)\left(X-\alpha_{j}^{\prime}\right)$ the minimal polynomial of $\alpha_{j}$. Since $\left|\xi-\alpha_{j}\right| \leq 1$ and $\left|\alpha_{j}-\alpha_{j}^{\prime}\right| \leq c$ for $j \geq c_{0}$, we have

$$
\max (1,|\xi|) \asymp \max \left(1,\left|\alpha_{j}\right|\right) \asymp \max \left(1,\left|\alpha_{j}^{\prime}\right|\right)
$$

for $j \geq c_{0}$. It follows from Lemma 3.14 that $H\left(P_{j}\right) \asymp\left|A_{j}\right|$ for $j \geq c_{0}$. By Lemma 3.15, we have $\left|\xi-\alpha_{j}\right|<\left|\alpha_{j}-\alpha_{j}^{\prime}\right|$ for $j \geq c_{0}$, which implies $\left|\xi-\alpha_{j}^{\prime}\right|=\left|\alpha_{j}-\alpha_{j}^{\prime}\right|$ for $j \geq c_{0}$. Hence, we have

$$
\left|P_{j}(\xi)\right| \asymp H\left(P_{j}\right)\left|\xi-\alpha_{j}\right|\left|\alpha_{j}-\alpha_{j}^{\prime}\right|
$$

for $j \geq c_{0}$. It follows from (24) and (25) that $w_{d}^{*}(\xi)+\varepsilon \leq w_{2}(\xi)$. Thus, we have $\varepsilon \leq$ $w_{n}(\xi)-w_{n}^{*}(\xi)$ for all $2 \leq n \leq d$.

Finally, we assume (21). By (25), we obtain

$$
\limsup _{j \rightarrow \infty} \frac{-\log \left|P_{j}(\xi)\right|}{\log H\left(P_{j}\right)} \leq w_{2}^{*}(\xi)+\chi
$$

Recall that $C_{1}(\alpha, C, d)$ is defined in Lemma 3.2. Put $c_{1}:=\max _{1 \leq i \leq d} C_{1}(\xi, 1, i)$. Let $P(X) \in\left(\mathbb{F}_{q}[T]\right)[X]_{\text {min }}$ be a polynomial of degree at most $d$ with $H(P) \geq c_{1}^{-\frac{1}{2}} H\left(\alpha_{c_{0}}\right)^{\frac{\delta}{2 \theta}}$ and $P\left(\alpha_{j}\right) \neq 0$ for all $j \geq 1$. We define an integer $j_{1} \geq c_{0}$ such that $H\left(\alpha_{j_{1}}\right) \leq\left(c_{1} H(P)^{2}\right)^{\frac{\theta+\iota}{\delta-\iota}}<$ $H\left(\alpha_{j_{1}+1}\right)$. Since

$$
H(P)<c_{1}^{-\frac{1}{2}} H\left(\alpha_{j_{1}+1}\right)^{\frac{\delta-\iota}{2(\theta+\iota)}} \leq c_{1}^{-\frac{1}{2}} H\left(\alpha_{j_{1}}\right)^{\frac{\delta-\iota}{2}},
$$

we have

$$
\left|P(\xi)-P\left(\alpha_{j_{1}}\right)\right| \leq c_{1} H(P)\left|\xi-\alpha_{j_{1}}\right|<H\left(\alpha_{j_{1}}\right)^{-d} H(P)^{-1} \leq\left|P\left(\alpha_{j_{1}}\right)\right|
$$

by Lemmas 3.2 and 3.16. Therefore, we obtain

$$
|P(\xi)|=\left|P\left(\alpha_{j_{1}}\right)\right| \geq H\left(\alpha_{j_{1}}\right)^{-d} H(P)^{-1} \geq c^{-\frac{d(\theta+\iota)}{\delta-\iota}} H(P)^{-1-\frac{2 d(\theta+\iota)}{\delta-\iota}} .
$$

Hence, we get

$$
w_{d}(\xi) \leq \max \left(w_{2}^{*}(\xi)+\chi, 1+\frac{2 d(\theta+\iota)}{\delta-\iota}\right)
$$

by Lemma 3.1. Since $\iota$ is arbitrarily small, we have $w_{d}(\xi) \leq w_{2}^{*}(\xi)+\chi$. This completes the proof.

\section{Proof of MAIN RESUlts}

Proof of Theorem 2.1. We take a strictly increasing sequence of positive integers $\left(k_{j}\right)_{j \geq 1}$, a sequence of integers $\left(n_{j}\right)_{j \geq 1}$, and a sequence of rational numbers $\left(u_{j}\right)_{j \geq 1}$ as in Lemma 
3.12, For $j \geq 1$, we put

$$
\begin{gathered}
d_{1, j}:=\frac{b_{j}^{n_{j}-2}(a-b)}{p^{m}}, \quad d_{i, j}:=\frac{a a_{j}^{i-2} b_{j}^{n_{j}-i-1}\left(a_{j}-b_{j}\right)}{p^{m}} \quad\left(2 \leq i \leq n_{j}-1\right), \\
d_{n_{j}, j}:=\frac{p^{k_{j}} b b_{j}^{n_{j}-2}-a a_{j}^{n_{j}-2}}{p^{m}} .
\end{gathered}
$$

Then we have $d_{i, j} \in \mathbb{Z}_{>0}$ and $\operatorname{gcd}\left(d_{n_{j}, j}, p\right)=1$ for all $j \geq 1,1 \leq i \leq n_{j}$. Now we take polynomials $A_{1, j}, \ldots, A_{n_{j}, j} \in \mathbb{F}_{q}[T]$ with $\operatorname{deg} A_{i, j}=d_{i, j}$. Put

$$
\xi_{j}:=\left[A_{1, j}, \ldots, A_{n_{j}, j}, A_{1, j}^{p^{k_{j}}}, \ldots, A_{n_{j}, j}^{p^{k_{j}}}, A_{1, j}^{p^{2 k_{j}}}, \ldots\right] \in \mathbb{F}_{q}\left(\left(T^{-1}\right)\right)
$$

and let $\left(p_{n, j} / q_{n, j}\right)_{n \geq 0}$ be the convergent sequence of $\xi_{j}$. By Theorem $3.8, \xi_{j}$ is of Class IA. Therefore, by Lemma 3.11, we deduce that $\xi_{j}$ is algebraic of degree $p^{k_{j}}+1$. For $1 \leq i \leq n_{j}$, we put

$$
r_{i, j}:=\frac{d_{i, j}}{p^{k_{j}}\left(\sum_{\ell=1}^{i-1} d_{\ell, j}\right)+\sum_{\ell=i}^{n_{j}} d_{\ell, j}} .
$$

Then a straightforward computation shows that

$$
\begin{gathered}
r_{1, j}=\frac{a-b}{\left(p^{k_{j}}-1\right) b}, \quad r_{i, j}=\frac{a_{j}-b_{j}}{\left(p^{k_{j}}-1\right) b_{j}} \quad\left(2 \leq i \leq n_{j}-1\right), \\
r_{n_{j}, j}=\frac{p^{k_{j}} b b_{j}^{n_{j}-2}-a a_{j}^{n_{j}-2}}{\left(p^{k_{j}}-1\right) a a_{j}^{n_{j}-2}} .
\end{gathered}
$$

By Theorem 3.9 and Lemma 3.12, we obtain

$$
\limsup _{n \rightarrow \infty} \frac{\operatorname{deg} q_{n+1, j}}{\operatorname{deg} q_{n, j}}=w, \quad \liminf _{n \rightarrow \infty} \frac{\operatorname{deg} q_{n+1, j}}{\operatorname{deg} q_{n, j}}>2 d-1 .
$$

It follows from Proposition 3.7 that

$$
w_{1}\left(\xi_{j}\right)=w_{1}^{*}\left(\xi_{j}\right)=\ldots=w_{d}\left(\xi_{j}\right)=w_{d}^{*}\left(\xi_{j}\right)=w .
$$

This completes the proof.

Proof of Theorem 2.6. Let $\left(\varepsilon_{n}\right)_{n \geq 1}$ be a sequence over the set $\{0,1\}$. We define recursively the sequences $\left(a_{n}\right)_{n \geq 0},\left(P_{n}\right)_{n \geq-1}$, and $\left(Q_{n}\right)_{n \geq-1}$ by

$$
\left\{\begin{array}{l}
a_{0}=0, a_{1}=T+\varepsilon_{1}, a_{n}=T^{\left\lfloor(w-1) \operatorname{deg} Q_{n-1}\right\rfloor}+\varepsilon_{n}, n \geq 2, \\
P_{-1}=1, P_{0}=0, P_{n}=a_{n} P_{n-1}+P_{n-2}, n \geq 1 \\
Q_{-1}=0, Q_{0}=1, Q_{n}=a_{n} Q_{n-1}+Q_{n-2}, n \geq 1 .
\end{array}\right.
$$

Set $\xi_{w}:=\left[0, a_{1}, a_{2}, \ldots\right]$. Then $\left(P_{n} / Q_{n}\right)_{n \geq 0}$ is the convergent sequence of $\xi_{w}$. It follows from Lemma 3.5 (iii) that

$$
\lim _{n \rightarrow \infty} \frac{\operatorname{deg} Q_{n+1}}{\operatorname{deg} Q_{n}}=w
$$

Therefore, by Proposition 3.7, we have

$$
w_{1}\left(\xi_{w}\right)=w_{1}^{*}\left(\xi_{w}\right)=\ldots=w_{d}\left(\xi_{w}\right)=w_{d}^{*}\left(\xi_{w}\right)=w .
$$

Proof of Theorem [2.8. For any $j \geq 1$, we put

$$
\xi_{w, j}:=\left[0, a_{1, w}, \ldots, a_{\left\lfloor w^{j}\right\rfloor, w}, \bar{a}\right] .
$$


Then $\xi_{w, j}$ is quadratic by Lemma 3.13. It follows from the proof of Theorem 1.1 in [25] that $\left(H\left(\xi_{w, j}\right)\right)_{j \geq 1}$ is a divergent increasing sequence, and

$$
\begin{gathered}
\lim _{j \rightarrow \infty} \frac{-\log \left|\xi_{w}-\xi_{w, j}\right|}{\log H\left(\xi_{w, j}\right)}=w, \quad \lim _{j \rightarrow \infty} \frac{-\log \left|\xi_{w, j}-\xi_{w, j}^{\prime}\right|}{\log H\left(\xi_{w, j}\right)}=1, \\
\limsup _{j \rightarrow \infty} \frac{\log H\left(\xi_{w, j+1}\right)}{\log H\left(\xi_{w, j}\right)} \leq w .
\end{gathered}
$$

By the definition of $w$, we have $2 d w \leq(w-2)(w-d)$. Applying Lemma 3.19 with $\delta=\rho=w-d, \varepsilon=\chi=1$, and $\theta=w$, we obtain (5) for all $2 \leq n \leq d$.

Proof of Theorem [2.9. For any $j \geq 1$, we put

$$
\xi_{w, \eta, j}:=\left[0, a_{1, w, \eta}, \ldots, a_{\left\lfloor w^{j}\right\rfloor, w, \eta}, \overline{a, \ldots, a, c}\right],
$$

where the length of the periodic part is $\left\lfloor\eta w^{j}\right\rfloor$. Then $\xi_{w, \eta, j}$ is quadratic by Lemma 3.13, It follows from the proof of Theorem 1.2 in [25] that $\left(H\left(\xi_{w, \eta, j}\right)\right)_{j \geq 1}$ is a divergent increasing sequence, and

$$
\begin{gathered}
\lim _{j \rightarrow \infty} \frac{-\log \left|\xi_{w, \eta}-\xi_{w, \eta, j}\right|}{\log H\left(\xi_{w, \eta, j}\right)}=\frac{2 w}{2+\eta}, \quad \lim _{j \rightarrow \infty} \frac{-\log \left|\xi_{w, \eta, j}-\xi_{w, \eta, j}^{\prime}\right|}{\log H\left(\xi_{w, \eta, j}\right)}=\frac{2}{2+\eta}, \\
\limsup _{j \rightarrow \infty} \frac{\log H\left(\xi_{w, \eta, j+1}\right)}{\log H\left(\xi_{w, \eta, j}\right)} \leq w .
\end{gathered}
$$

A direct computation shows that

$$
2 d w \leq\left(\frac{2 w}{2+\eta}-2\right)\left(\frac{2 w}{2+\eta}-d\right) .
$$

Applying Lemma 3.19 with

$$
\delta=\rho=\frac{2 w}{2+\eta}-d, \quad \varepsilon=\chi=\frac{2}{2+\eta}, \quad \theta=w,
$$

we have (6) for all $2 \leq n \leq d$.

\section{FURThER REMARKS}

In this section, we give some theorems associated to the main results.

5.1. Relationship between automatic sequences and Diophantine exponents. Let $k \geq 2$ be an integer. We denote by $\Sigma_{k}$ the set $\{0,1, \ldots, k-1\}$. A $k$-automaton is defined to be a sextuple

$$
A=\left(Q, \Sigma_{k}, \delta, q_{0}, \Delta, \tau\right)
$$

where $Q$ is a finite set of states, $\delta: Q \times \Sigma_{k} \rightarrow Q$ is a transition function, $q_{0} \in Q$ is an initial state, a finite set $\Delta$ is an output alphabet, and $\tau: Q \rightarrow \Delta$ is an output function. For $q \in Q$ and a finite word $W=w_{0} w_{1} \cdots w_{n}$ over $\Sigma_{k}$, we define $\delta(q, W)$ recursively by $\delta(q, W)=\delta\left(\delta\left(q, w_{0} w_{1} \cdots w_{n-1}\right), w_{n}\right)$. For an integer $n \geq 0$, we put $W_{n}:=w_{r} w_{r-1} \cdots w_{0}$, where $\sum_{i=0}^{r} w_{i} k^{i}$ is the $k$-ary expansion of $n$. An infinite sequence $\left(a_{n}\right)_{n \geq 0}$ is said to be $k$ automatic if there exists a $k$-automaton $A=\left(Q, \Sigma_{k}, \delta, q_{0}, \Delta, \tau\right)$ such that $a_{n}=\tau\left(\delta\left(q_{0}, W_{n}\right)\right)$ for all $n \geq 0$.

Christol, Kamae, Mendes France, and Rauzy [16] characterized algebraic Laurent series by using finite automatons. More precisely, they showed that for a sequence $\left(a_{n}\right)_{n \geq 0}$ over $\mathbb{F}_{q}$, the Laurent series $\sum_{n=0}^{\infty} a_{n} T^{-n}$ is algebraic if and only if $\left(a_{n}\right)_{n \geq 0}$ is $p$-automatic. It is known that for $m \geq 1$ and $k \geq 2$, a sequence $\left(a_{n}\right)_{n \geq 0}$ is $k$-automatic if and only if it is $k^{m}$-automatic (see Theorem 6.6.4 in [1]). Therefore, we obtain the following corollary of Theorem 2.1. 
Corollary 5.1. Let $d, m \geq 1$ be integers and $w>2 d-1$ be a rational number. Then there exists a $p^{m}$-automatic sequence $\left(a_{n}\right)_{n \geq 0}$ over $\mathbb{F}_{q}$ such that

$$
w_{1}(\xi)=w_{1}^{*}(\xi)=\ldots=w_{d}(\xi)=w_{d}^{*}(\xi)=w,
$$

where $\xi=\sum_{n=0}^{\infty} a_{n} T^{-n}$.

In this subsection, we consider the problem of determining whether or not we can extend Corollary 5.1 to $k$-automatic sequence for any integer $k \geq 2$. It is the natural problem in view of Corollary 5.1 .

Let $k \geq 2$ be an integer. We define a set $S_{k}$ of rational numbers as follows:

$$
S_{k}=\left\{\frac{k^{a}}{\ell} \mid a, \ell \in \mathbb{Z}_{\geq 1}\right\} .
$$

Bugeaud [6] proved that for an integer $k \geq 2$ and $w \in S_{k}$ with $w>2$, there exists a $k$ automatic sequence $\left(a_{n}\right)_{n \geq 0}$ over $\{0,2\}$ such that $w_{1}(\xi)=w-1$, where $\xi=\sum_{n=0}^{\infty} a_{n} / 3^{n}$. The proof of this result essentially depends on the Folding Lemma and an analogue of Lemma 3.6. It is known that the Folding Lemma holds for Laurent series over a finite field. For the statement and proof of the Folding Lemma, we refer the readers to [28, Proposition 2] and [31, the proof of Theorem 1]. We have the following theorem which is similar to Bugeaud's result.

Theorem 5.2. Let $k \geq 2$ be an integer and $w>2$ be in $S_{k}$. Then there exists a $k$ automatic sequence $\left(a_{n}\right)_{n \geq 0}$ over $\mathbb{F}_{q}$ such that $w_{1}(\xi)=w-1$, where $\xi=\sum_{n=0}^{\infty} a_{n} T^{-n}$.

Using Lemma 3.4, we prove the following theorem.

Theorem 5.3. Let $d \geq 1$ be an integer, $k \geq 2$ be an integer, and $w>\left(2 d+1+\sqrt{4 d^{2}+1}\right) / 2$ be in $S_{k}$. Then there exists a $k$-automatic sequence $\left(a_{n}\right)_{n \geq 0}$ over $\mathbb{F}_{q}$ such that

$$
w_{1}(\xi)=w_{1}^{*}(\xi)=\ldots=w_{d}(\xi)=w_{d}^{*}(\xi)=w-1,
$$

where $\xi=\sum_{n=0}^{\infty} a_{n} T^{-n}$.

Note that $\left(2 d-1+\sqrt{4 d^{2}+1}\right) / 2$ is greater than $2 d-1$ for any $d \geq 1$.

Proof. Slightly modifying the proof of Theorem 1.2 in [12, we deduce that there exists a $k$-automatic sequence $\left(a_{n}\right)_{n \geq 0}$ with $a_{n} \in\{0,1\}$ for all $n \geq 1$ which satisfies the following properties:

- $\frac{2 d+1+\sqrt{4 d^{2}+1}}{2}<\frac{n_{j+1}}{n_{j}} \leq w$ holds for all $j \geq 0$,

- there exist infinitely many $j \geq 0$ such that $\frac{n_{j+1}}{n_{j}}=w$,

where

$$
\left\{n \in \mathbb{Z}_{\geq 0} \mid a_{n}=1\right\}=:\left\{n_{0}<n_{1}<n_{2}<\ldots\right\} .
$$

We put $q_{j}:=T^{n_{j}}$ and $p_{j}:=1+T^{n_{j}-n_{j-1}}+\cdots+T^{n_{j}-n_{0}}$ for any $j \geq 0$. Then we have for any $j \geq 0, \operatorname{gcd}\left(p_{j}, q_{j}\right)=1$ and $p_{j} / q_{j}=\sum_{n=0}^{n_{j}} a_{n} T^{-n}$. We put $\xi:=\sum_{n=0}^{\infty} a_{n} T^{-n}$. A direct computation shows that

$$
\frac{-\log \left|\xi-p_{n_{j}} / q_{n_{j}}\right|}{\log \left|q_{n_{j}}\right|}=\frac{\log \left|q_{n_{j+1}}\right|}{\log \left|q_{n_{j}}\right|}=\frac{n_{j+1}}{n_{j}}
$$

for any $j \geq 0$. By the definition of $w_{1}$, we obtain $w_{1}(\xi) \geq w-1$. Applying Lemma 3.4 with $\theta=w, \rho=w-d$, and $\delta=\left(1+\sqrt{4 d^{2}+1}\right) / 2$, we deduce that

$$
w_{1}(\xi)=w_{1}^{*}(\xi)=\ldots=w_{d}(\xi)=w_{d}^{*}(\xi)=w-1 .
$$


5.2. Analogues of Theorems 2.8 and 2.9 for real and $p$-adic numbers. In this subsection, we give analogues of Theorems 2.8 and 2.9, which are generalizations of Theorems 4.1 and 4.2 in [10], and Theorems 1 and 2 in [11].

Theorem 5.4. Let $d \geq 2$ be an integer and $w \geq\left(3 d+2+\sqrt{9 d^{2}+4 d+4}\right) / 2$ be a real number. Let $a, b$ be distinct positive integers. Let $\left(a_{n, w}\right)_{n \geq 1}$ be a sequence given by

$$
a_{n, w}= \begin{cases}b & \text { if } n=\left\lfloor w^{i}\right\rfloor \text { for some integer } i \geq 0, \\ a & \text { otherwise. }\end{cases}
$$

Set the continued fraction $\xi_{w}:=\left[0, a_{1, w}, a_{2, w}, \ldots\right] \in \mathbb{R}$. Then we have

$$
w_{n}^{*}\left(\xi_{w}\right)=w-1, \quad w_{n}\left(\xi_{w}\right)=w
$$

for all $2 \leq n \leq d$.

Theorem 5.5. Let $d \geq 2$ be an integer, $w \geq 121 d^{2}$ be a real number, and $a, b, c$ be distinct positive integers. Let $0<\eta<\sqrt{w} / d$ be a positive number and put $m_{i}:=\left\lfloor\left(\left\lfloor w^{i+1}\right\rfloor-\left\lfloor w^{i}-\right.\right.\right.$ $\left.1\rfloor) /\left\lfloor\eta w^{i}\right\rfloor\right\rfloor$ for all $i \geq 1$. Let $\left(a_{n, w, \eta}\right)_{n \geq 1}$ be the sequence given by

$$
a_{n, w, \eta}= \begin{cases}b & \text { if } n=\left\lfloor w^{i}\right\rfloor \text { for some integer } i \geq 0, \\ c & \text { if } n \neq\left\lfloor w^{i}\right\rfloor \text { for all integers } i \geq 0, \text { and } n=\left\lfloor w^{j}\right\rfloor+ \\ & m\left\lfloor\eta w^{j}\right\rfloor \text { for some integers } 1 \leq m \leq m_{j}, j \geq 1, \\ a & \text { otherwise. }\end{cases}
$$

Set the continued fraction $\xi_{w, \eta}:=\left[0, a_{1, w, \eta}, a_{2, w, \eta}, \ldots\right] \in \mathbb{R}$. Then we have

$$
w_{n}^{*}\left(\xi_{w, \eta}\right)=\frac{2 w-2-\eta}{2+\eta}, \quad w_{n}\left(\xi_{w, \eta}\right)=\frac{2 w-\eta}{2+\eta}
$$

for all $2 \leq n \leq d$. Hence, we have

$$
w_{n}\left(\xi_{w, \eta}\right)-w_{n}^{*}\left(\xi_{w, \eta}\right)=\frac{2}{2+\eta}
$$

for all $2 \leq n \leq d$.

It seems that for each $d \geq 3$, the real numbers $\xi$ defined by Theorems 5.4 and 5.5 are the first explicit continued fraction examples for which $w_{d}(\xi)$ and $w_{d}^{*}(\xi)$ are different.

We denote by $|\cdot|_{p}$ the absolute value of $\mathbb{Q}_{p}$ normalized to satisfy $|p|_{p}=p^{-1}$. We recall the definitions of Diophantine exponents $w_{n}$ and $w_{n}^{*}$ in $\mathbb{Q}_{p}$. For $\xi \in \mathbb{Q}_{p}$ and an integer $n \geq 1$, we denote by $w_{n}(\xi)$ (resp. $\left.w_{n}^{*}(\xi)\right)$ the supremum of the real numbers $w$ (resp. $w^{*}$ ) which satisfy

$$
0<|P(\xi)|_{p} \leq H(P)^{-w-1} \quad\left(\text { resp. } 0<|\xi-\alpha|_{p} \leq H(\alpha)^{-w^{*}-1}\right)
$$

for infinitely many $P(X) \in \mathbb{Z}[X]$ of degree at most $n$ (resp. algebraic numbers $\alpha \in \mathbb{Q}_{p}$ of degree at most $n$ ).

Theorem 5.6. Let $d \geq 2$ be an integer and $w \geq\left(3 d+2+\sqrt{9 d^{2}+4 d+4}\right) / 2$ be a real number. Let $b$ be a positive integer and $\left(\varepsilon_{j}\right)_{j \geq 0}$ be a sequence in $\{0,1\}$. Let $\left(a_{n, w}\right)_{n \geq 1}$ be a sequence given by

$$
a_{n, w}= \begin{cases}b+3 i+2 & \text { if } n=\left\lfloor w^{i}\right\rfloor \text { for some integer } i \geq 0, \\ b+3 i+\varepsilon_{j} & \text { if }\left\lfloor w^{i}\right\rfloor<n<\left\lfloor w^{i+1}\right\rfloor \text { for some integer } i \geq 0 .\end{cases}
$$

Set the Schneider's p-adic continued fraction $\xi_{w}:=\left[a_{1, w}, a_{2, w}, \ldots\right] \in \mathbb{Q}_{p}$. Then we have

$$
w_{n}^{*}\left(\xi_{w}\right)=w-1, \quad w_{n}\left(\xi_{w}\right)=w
$$

for all $2 \leq n \leq d$. 
Theorem 5.7. Let $d \geq 2$ be an integer and $w \geq 121 d^{2}$ be a real number. Let $b$ be positive integer, $\left(\varepsilon_{j}\right)_{j \geq 0}$ be a sequence in $\{0,1\}$, and $0<\eta<\sqrt{w} / d$ be a positive number. Let $\left(a_{n, w, \eta}\right)_{n \geq 1}$ be the sequence given by

$$
a_{n, w, \eta}= \begin{cases}b+4 i+3 & \text { if } n=\left\lfloor w^{i}\right\rfloor \text { for some integer } i \geq 0, \\ b+4 i+2 & \text { if }\left\lfloor w^{i}\right\rfloor<n<\left\lfloor w^{i+1}\right\rfloor \text { for some integer } i \geq \\ & 0 \text { and }\left(n-\left\lfloor w^{i}\right\rfloor\right) /\left\lfloor\eta w^{i}\right\rfloor \in \mathbb{Z}, \\ b+4 i+\varepsilon_{i} & \text { if }\left\lfloor w^{i}\right\rfloor<n<\left\lfloor w^{i+1}\right\rfloor \text { for some integer } i \geq \\ & 0 \text { and }\left(n-\left\lfloor w^{i}\right\rfloor\right) /\left\lfloor\eta w^{i}\right\rfloor \notin \mathbb{Z} .\end{cases}
$$

Set the Schneider's p-adic continued fraction $\xi_{w, \eta}:=\left[a_{1, w, \eta}, a_{2, w, \eta}, \ldots\right] \in \mathbb{Q}_{p}$. Then we have

$$
w_{n}^{*}\left(\xi_{w, \eta}\right)=\frac{2 w-2-\eta}{2+\eta}, \quad w_{n}\left(\xi_{w, \eta}\right)=\frac{2 w-\eta}{2+\eta}
$$

for all $2 \leq n \leq d$. Hence, we have

$$
w_{n}\left(\xi_{w, \eta}\right)-w_{n}^{*}\left(\xi_{w, \eta}\right)=\frac{2}{2+\eta}
$$

for all $2 \leq n \leq d$.

The definition and notation of the Schneider's $p$-adic continued fractions can be found in 11. It seems that for each $d \geq 3$, the $p$-adic numbers $\xi$ defined by Theorems 5.6 and 5.7 are the first explicit continued fraction examples for which $w_{d}(\xi)$ and $w_{d}^{*}(\xi)$ are different.

In what follows, we prepare lemmas in order to prove the above theorems. We omit the details of proofs of these lemmas.

We denote by $\mathbb{Z}[X]_{\min }$ the set of all non-constant, irreducible, primitive polynomials in $\mathbb{Z}[X]$ whose leading coefficients are positive. For $\xi \in \mathbb{R}$ and an integer $n \geq 1$, we denote by $\tilde{w}_{n}(\xi)$ the supremum of the real numbers $w$ which satisfy

$$
0<|P(\xi)| \leq H(P)^{-w}
$$

for infinitely many $P(X) \in \mathbb{Z}[X]_{\min }$ of degree at most $n$. For $\xi \in \mathbb{Q}_{p}$ and an integer $n \geq 1$, we denote by $\tilde{w}_{n}(\xi)$ the supremum of the real numbers $w$ which satisfy

$$
0<|P(\xi)|_{p} \leq H(P)^{-w-1}
$$

for infinitely many $P(X) \in \mathbb{Z}[X]_{\min }$ of degree at most $n$.

Using Gelfond's Lemma (see Lemma A.3 in 4) instead of (17), we obtain an analogue of Lemma 3.1 for real numbers.

Lemma 5.8. Let $n \geq 1$ be an integer and $\xi$ be a real number. Then we have

$$
w_{n}(\xi)=\tilde{w}_{n}(\xi) .
$$

Using Gelfond's Lemma and the fact that $|a||a|_{p} \geq 1$ for non-zero integers $a$, we obtain an analogue of Lemma 3.1 for $p$-adic numbers.

Lemma 5.9. Let $n \geq 1$ be an integer and $\xi$ be in $\mathbb{Q}_{p}$. Then we have

$$
w_{n}(\xi)=\tilde{w}_{n}(\xi) .
$$

Analogues of Lemma 3.2 for real and $p$-adic numbers follow in a similar way to the proof of Lemma 3.2 .

Lemma 5.10. Let $\alpha, \beta$ be real numbers and $P(X) \in \mathbb{Z}[X]$ be a non-constant polynomial of degree d. Let $C \geq 0$ be a real number. Assume that $|\alpha-\beta| \leq C$. Then there exists a positive constant $C_{2}(\alpha, C, d)$, depending only on $\alpha, C$, and $d$ such that

$$
|P(\alpha)-P(\beta)| \leq C_{2}(\alpha, C, d)|\alpha-\beta| H(P) .
$$


Lemma 5.11. Let $\alpha, \beta$ be in $\mathbb{Q}_{p}$ and $P(X) \in \mathbb{Z}[X]$ be a non-constant polynomial of degree d. Let $C \geq 0$ be a real number. Assume that $|\alpha-\beta|_{p} \leq C$. Then there exists a positive constant $C_{3}(\alpha, C, d)$, depending only on $\alpha, C$, and $d$ such that

$$
|P(\alpha)-P(\beta)|_{p} \leq C_{3}(\alpha, C, d)|\alpha-\beta|_{p} .
$$

Lemmas 3.1, 3.2, 3.14 (16), and 3.15, 3.17 are used in the proof of Lemma 3.19, Analogues of Lemmas 3.15 3.17for real numbers and Lemmas 3.15 and 3.17for $p$-adic numbers are already known (see p.730 in [10], Theorem A.1 and Corollary A.2 in [4], and Lemmas 3.2 and 2.5 in [26]). Slightly modifying the proof of Lemma 2.5 in [26], we obtain an analogue of Lemma 3.16 for $p$-adic numbers. That is, for non-constant polynomial $P(X) \in \mathbb{Z}[X]$ of degree $m$ and algebraic number $\alpha \in \mathbb{Q}_{p}$ of degree $n$,

$$
P(\alpha)=0 \text { or }|P(\alpha)|_{p} \geq c H(P)^{-n} H(\alpha)^{-m},
$$

where $c$ is a positive constant depending only on $m$ and $n$. The inequality (30) is probably known. However, we were unable to find it in the literature.

Using Lemma A.2 in [4] instead of (16), in addition to analogues of 3.1, 3.2, and 3.153.17, we obtain an analogue of Lemma 3.19 for real numbers.

Lemma 5.12. Let $d \geq 2$ be an integer. Let $\xi$ be a real number, and $\theta, \rho, \delta$ be positive numbers. Assume that there exists a sequence $\left(\alpha_{j}\right)_{j \geq 1}$ such that for any $j \geq 1, \alpha_{j} \in \mathbb{R}$ is quadratic, $\left(H\left(\alpha_{j}\right)\right)_{j \geq 1}$ is a divergent increasing sequence, and

$$
\begin{gathered}
\limsup _{j \rightarrow \infty} \frac{\log H\left(\alpha_{j+1}\right)}{\log H\left(\alpha_{j}\right)} \leq \theta, \\
d+\delta \leq \liminf _{j \rightarrow \infty} \frac{-\log \left|\xi-\alpha_{j}\right|}{\log H\left(\alpha_{j}\right)}, \quad \limsup _{j \rightarrow \infty} \frac{-\log \left|\xi-\alpha_{j}\right|}{\log H\left(\alpha_{j}\right)} \leq d+\rho .
\end{gathered}
$$

If $2 d \theta \leq(d-2+\rho) \delta$, then we have for all $2 \leq n \leq d$,

$$
d-1+\delta \leq w_{n}^{*}(\xi) \leq d-1+\rho .
$$

Furthermore, assume that there exist a non-negative number $\varepsilon$ and a positive number $c$ such that for any $j \geq 1,0<\left|\alpha_{j}-\alpha_{j}^{\prime}\right| \leq c$ and

$$
\limsup _{j \rightarrow \infty} \frac{-\log \left|\alpha_{j}-\alpha_{j}^{\prime}\right|}{\log H\left(\alpha_{j}\right)} \geq \varepsilon .
$$

If $2 d \theta \leq(d-2+\delta) \delta$, then we have for all $2 \leq n \leq d$,

$$
d-1+\delta \leq w_{n}^{*}(\xi) \leq d-1+\rho, \quad \varepsilon \leq w_{n}(\xi)-w_{n}^{*}(\xi) .
$$

Finally, assume that there exists a non-negative number $\chi$ such that

$$
\limsup _{i \rightarrow \infty} \frac{-\log \left|\alpha_{i}-\alpha_{i}^{\prime}\right|}{\log H\left(\alpha_{i}\right)} \leq \chi .
$$

Then we have for all $2 \leq n \leq d$,

$$
d-1+\delta \leq w_{n}^{*}(\xi) \leq d-1+\rho, \quad \varepsilon \leq w_{n}(\xi)-w_{n}^{*}(\xi) \leq \chi .
$$

Lemma 5.13. Let $d \geq 2$ be an integer. Let $\xi$ be in $\mathbb{Q}_{p}$ with $|\xi|_{p} \leq 1$ and $\theta, \rho, \delta$ be positive numbers. Assume that there exists a sequence $\left(\alpha_{j}\right)_{j \geq 1}$ such that for any $j \geq 1, \alpha_{j} \in \mathbb{Q}_{p}$ is quadratic, $\left(H\left(\alpha_{j}\right)\right)_{j \geq 1}$ is a divergent increasing sequence, and

$$
\begin{gathered}
\limsup _{j \rightarrow \infty} \frac{\log H\left(\alpha_{j+1}\right)}{\log H\left(\alpha_{j}\right)} \leq \theta, \\
d+\delta \leq \liminf _{j \rightarrow \infty} \frac{-\log \left|\xi-\alpha_{j}\right|_{p}}{\log H\left(\alpha_{j}\right)}, \quad \limsup _{j \rightarrow \infty} \frac{-\log \left|\xi-\alpha_{j}\right|_{p}}{\log H\left(\alpha_{j}\right)} \leq d+\rho .
\end{gathered}
$$


If $2 d \theta \leq(d-2+\rho) \delta$, then we have for all $2 \leq n \leq d$,

$$
d-1+\delta \leq w_{n}^{*}(\xi) \leq d-1+\rho .
$$

Furthermore, assume that there exists a non-negative number $\varepsilon$ such that for any $j \geq$ $1,0<\left|\alpha_{j}-\alpha_{j}^{\prime}\right|_{p} \leq 1$ and

$$
\limsup _{j \rightarrow \infty} \frac{-\log \left|\alpha_{j}-\alpha_{j}^{\prime}\right|_{p}}{\log H\left(\alpha_{j}\right)} \geq \varepsilon
$$

If $2 d \theta \leq(d-2+\delta) \delta$, then we have for all $2 \leq n \leq d$,

$$
d-1+\delta \leq w_{n}^{*}(\xi) \leq d-1+\rho, \quad \varepsilon \leq w_{n}(\xi)-w_{n}^{*}(\xi) .
$$

Finally, assume that there exists a non-negative number $\chi$ such that

$$
\limsup _{i \rightarrow \infty} \frac{-\log \left|\alpha_{i}-\alpha_{i}^{\prime}\right|_{p}}{\log H\left(\alpha_{i}\right)} \leq \chi .
$$

Then we have for all $2 \leq n \leq d$,

$$
d-1+\delta \leq w_{n}^{*}(\xi) \leq d-1+\rho, \quad \varepsilon \leq w_{n}(\xi)-w_{n}^{*}(\xi) \leq \chi .
$$

Note that the proof of Lemma 5.13 uses analogues of Lemmas 3.1, 3.2, and 3.15 3.17 and the following: Let $P_{j}(X)=A_{j}\left(X-\alpha_{j}\right)\left(X-\alpha_{j}^{\prime}\right) \in \mathbb{Z}[X]_{\text {min }}$ be the minimal polynomial of $\alpha_{j}$. Then, by the proof of Lemma 5 in [11], we have $\left|A_{j}\right|_{p}=1$ for sufficiently large $j$.

Proof of Theorem 5.4. For any $j \geq 2$, we put

$$
\xi_{w, j}:=\left[0, a_{1, w}, \ldots, a_{\left\lfloor w^{j}\right\rfloor, w}, \bar{a}\right] \in \mathbb{R} .
$$

It follows from the proof of Theorem 4.1 in $[8]$ that $\xi_{w, j}$ are quadratic irrationals, $\left(H\left(\xi_{w, j}\right)\right)_{j \geq 1}$ is a divergent increasing sequence, and

$$
\begin{gathered}
\lim _{j \rightarrow \infty} \frac{-\log \left|\xi_{w}-\xi_{w, j}\right|}{\log H\left(\xi_{w, j}\right)}=w, \quad \lim _{j \rightarrow \infty} \frac{-\log \left|\xi_{w, j}-\xi_{w, j}^{\prime}\right|}{\log H\left(\xi_{w, j}\right)}=1, \\
\limsup _{j \rightarrow \infty} \frac{\log H\left(\xi_{w, j+1}\right)}{\log H\left(\xi_{w, j}\right)} \leq w .
\end{gathered}
$$

Hence, we have (26) by Lemma 5.12 .

Proof of Theorem 5.5. For any $j \geq 2$, we put

$$
\xi_{w, \eta, j}:=\left[0, a_{1, w, \eta}, \ldots, a_{\left\lfloor w^{j}\right\rfloor, w, \eta}, \overline{a, \ldots, a, c}\right] \in \mathbb{R},
$$

where the length of the periodic part is $\left\lfloor\eta w^{j}\right\rfloor$. It follows from the proof of Theorem 4.3 in [8] that $\xi_{w, \eta, j}$ are quadratic irrationals, $\left(H\left(\xi_{w, \eta, j}\right)\right)_{j \geq 1}$ is a divergent increasing sequence, and

$$
\begin{gathered}
\lim _{j \rightarrow \infty} \frac{-\log \left|\xi_{w, \eta}-\xi_{w, \eta, j}\right|}{\log H\left(\xi_{w, \eta, j}\right)}=\frac{2 w}{2+\eta}, \quad \lim _{j \rightarrow \infty} \frac{-\log \left|\xi_{w, \eta, j}-\xi_{w, \eta, j}^{\prime}\right|}{\log H\left(\xi_{w, \eta, j}\right)}=\frac{2}{2+\eta}, \\
\limsup _{j \rightarrow \infty} \frac{\log H\left(\xi_{w, \eta, j+1}\right)}{\log H\left(\xi_{w, \eta, j}\right)} \leq w .
\end{gathered}
$$

Hence, we have (27) by Lemma 5.12 .

Proof of Theorem 5.6. For any $j \geq 2$, we put

$$
\xi_{w, j}:=\left[a_{1, w}, \ldots, a_{\left\lfloor w^{j}\right\rfloor, w}, \overline{a_{\left\lfloor w^{j}\right\rfloor+1, w}}\right] \in \mathbb{Q}_{p} .
$$


It follows from the proof of Theorem 1 in [11] that $\xi_{w, j}$ are quadratic irrationals, $\left(H\left(\xi_{w, j}\right)\right)_{j \geq 1}$ is a divergent increasing sequence, and

$$
\begin{gathered}
\lim _{j \rightarrow \infty} \frac{-\log \left|\xi_{w}-\xi_{w, j}\right|_{p}}{\log H\left(\xi_{w, j}\right)}=w, \quad \lim _{j \rightarrow \infty} \frac{-\log \left|\xi_{w, j}-\xi_{w, j}^{\prime}\right|_{p}}{\log H\left(\xi_{w, j}\right)}=1, \\
\limsup _{j \rightarrow \infty} \frac{\log H\left(\xi_{w, j+1}\right)}{\log H\left(\xi_{w, j}\right)} \leq w .
\end{gathered}
$$

Hence, we have (28) by Lemma 5.13 .

Proof of Theorem 5.7. For any $j \geq 2$, we put

$$
\xi_{w, \eta, j}:=\left[a_{1, w, \eta}, \ldots, a_{\left\lfloor w^{j}\right\rfloor, w, \eta}, \overline{a_{\left\lfloor w^{j}\right\rfloor+1, w, \eta}, \ldots, a_{\left\lfloor w^{j}\right\rfloor+\left\lfloor\eta w^{j}\right\rfloor, w, \eta}}\right] \in \mathbb{Q}_{p} .
$$

It follows from the proof of Theorem 2 in [11] that $\xi_{w, \eta, j}$ are quadratic irrationals, $\left(H\left(\xi_{w, \eta, j}\right)\right)_{j \geq 1}$ is a divergent increasing sequence, and

$$
\begin{gathered}
\lim _{j \rightarrow \infty} \frac{-\log \left|\xi_{w, \eta}-\xi_{w, \eta, j}\right|_{p}}{\log H\left(\xi_{w, \eta, j}\right)}=\frac{2 w}{2+\eta}, \quad \lim _{j \rightarrow \infty} \frac{-\log \left|\xi_{w, \eta, j}-\xi_{w, \eta, j}^{\prime}\right|_{p}}{\log H\left(\xi_{w, \eta, j}\right)}=\frac{2}{2+\eta}, \\
\limsup _{j \rightarrow \infty} \frac{\log H\left(\xi_{w, \eta, j+1}\right)}{\log H\left(\xi_{w, \eta, j}\right)} \leq w .
\end{gathered}
$$

Hence, we have (29) by Lemma 5.13 .

Acknowledgements. The author would like to express his gratitude to Prof. Shigeki Akiyama for the helpful comments on Lemma 3.12 and improving the language of this paper. The author is deeply grateful to Prof. Hajime Kaneko for the helpful comments on Corollary 2.5 and Lemma 3.12. The author also wishes to express his thanks to Prof. Dinesh S. Thakur for several helpful comments. The author would like to thank the referee for careful reading of this paper and giving helpful comments.

\section{REFERENCES}

[1] J.-P. Allouche, J. Shallit, Automatic Sequences: Theory, Applications, Generalizations, Cambridge University Press, Cambridge (2003).

[2] M. Amou, Approximation to certain transcendental decimal fractions by algebraic numbers, J. Number Theory 37 (1991), no. 2, 231-241.

[3] R. C. Baker, On approximation with algebraic numbers of bounded degree, Mathematika 23 (1976), no. $1,18-31$.

[4] Y. Bugeaud, Approximation by algebraic numbers, Cambridge Tracts in Mathematics, 160 Cambridge University Press, Cambridge, 2004.

[5] Y. Bugeaud, Mahler's classification of numbers compared with Koksma's. III, Publ. Math. Debrecen 65 (2004), no. 3-4, 305-316.

[6] Y. Bugeaud, Diophantine approximation and Cantor sets, Math. Ann. 341 (2008), no. 3, 677-684.

[7] Y. Bugeaud, On simultaneous rational approximation to a real number and its integral powers, Ann. Inst. Fourier (Grenoble) 60 (2010), no. 6, 2165-2182.

[8] Y. Bugeaud, A. Dujella, Root separation for irreducible integer polynomials, Bull. Lond. Math. Soc. 43 (2011), no. 6, 1239-1244.

[9] Y. Bugeaud, N. Budarina, D. Dickinson, H. O'Donnell, On simultaneous rational approximation to a p-adic number and its integral powers, Proc. Edinb. Math. Soc. (2) 54 (2011), no. 3, 599-612.

[10] Y. Bugeaud, Continued fractions with low complexity: transcendence measures and quadratic approximation, Compos. Math. 148 (2012), no. 3, 718-750.

[11] Y. Bugeaud, T. Pejković, Quadratic approximation in $\mathbb{Q}_{p}$, Int. J. Number Theory 11 (2015), no. 1, 193-209.

[12] Y. Bugeaud, Quadratic approximation to automatic continued fractions, J. Théor. Nombres Bordeaux 27 (2015), no. 2, 463-482.

[13] J. W. S. Cassels, Local fields, London Mathematical Society Student Texts, 3 Cambridge University Press, Cambridge, (1986). 
[14] T. Chaichana, V. Laohakosol, A. Harnchoowong, Linear independence of continued fractions in the field of formal series over a finite field, Thai J. Math. 4 (2006), no. 1, 163-177.

[15] H-J. Chen, Distribution of Diophantine approximation exponents for algebraic quantities in finite characteristic, J. Number Theory 133 (2013), no. 11, 3620-3644.

[16] G. Christol, T. Kamae, M. Mendes France, G. Rauzy, Suites algébriques, automates et substitutions, (French) Bull. Soc. Math. France 108 (1980), no. 4, 401-419.

[17] A. Firicel, Rational approximations to algebraic Laurent series with coefficients in a finite field, Acta Arith. 157 (2013), no. 4, 297-322.

[18] J. F. Koksma, Über die Mahlersche Klasseneinteilung der transzendenten Zahlen und die Approximation komplexer Zahlen durch algebraische Zahlen, (German) Monatsh. Math. Phys. 48, (1939), $176-189$.

[19] A. Lasjaunias, A survey of Diophantine approximation in fields of power series, Monatsh. Math. 130 (2000), no. 3, 211-229.

[20] K. Mahler, Zur Approximation der Exponentialfunktionen und des Logarithmus, I, II, (German) J. reine angew. Math., 166, (1932), 118-150.

[21] K. Mahler, On a theorem of Liouville in fields of positive characteristic, Canadian J. Math. 1, (1949), 397-400.

[22] B. de Mathan, Approximations diophantiennes dans un corps local, (French) Bull. Soc. Math. France Suppl. Mém. 21 (1970), 93pp.

[23] B. de Mathan, Approximation exponents for algebraic functions in positive characteristic, Acta Arith. 60 (1992), no. 4, 359-370.

[24] R. Müller, Algebraische Unabhängigkeit der Werte gewisser Lückenreihen in nicht-archimedisch bewerteten Körpern, (German) Results Math., 24, (1993), no. 3-4, 288-297.

[25] T. Ooto, Quadratic approximation in $\mathbb{F}_{q}\left(\left(T^{-1}\right)\right)$, Osaka J. Math. 54 (2017), no. 1, 129-156.

[26] T. Pejković, Polynomial root separation and applications, $\mathrm{PhD}$ Thesis, Université de Strasbourg and University of Zagreb, Strasbourg, 2012.

[27] N. Popescu, A. Zaharescu, On the structure of the irreducible polynomials over local fields, J. Number Theory 52 (1995), no. 1, 98-118.

[28] A. J. van der Poorten, J. Shallit, Folded continued fractions, J. Number Theory 40 (1992), no. 2, $237-250$.

[29] K. F. Roth, Rational approximations to algebraic numbers, Mathematika 2 (1955), 1-20.

[30] W. M. Schmidt, On continued fractions and Diophantine approximation in power series fields, Acta Arith. 95 (2000), no. 2, 139-166.

[31] J. Shallit, Simple continued fractions for some irrational numbers, J. Number Theory 11 (1979), 209-217.

[32] V. G. Sprindžuk, Mahler's problem in metric number theory, Izdat. gNauka iTehnikah, Minsk, (Russian). English translation by B. Volkmann, Translations of Mathematical Monographs, 25, American Mathematical Society, Providence, RI, 1969.

[33] D. S. Thakur, Diophantine approximation exponents and continued fractions for algebraic power series, J. Number Theory 79 (1999), no. 2, 284-291.

[34] D. S. Thakur, Higher Diophantine approximation exponents and continued fraction symmetries for function fields, Proc. Amer. Math. Soc. 139 (2011), no. 1, 11-19.

[35] D. S. Thakur, Higher Diophantine approximation exponents and continued fraction symmetries for function fields II, Proc. Amer. Math. Soc. 141 (2013), no. 8, 2603-2608.

Graduate School of Pure and Applied Sciences, University of Tsukuba, Tennodai 1-1-1, TSUKUBA, IBARAKI, 305-8571, JAPAN

E-mail address: ooto@math.tsukuba.ac.jp 